\title{
Dissecting the functions of SMG5, SMG7, and PNRC2 in nonsense-mediated mRNA decay of human cells
}

\author{
PAMELA NICHOLSON, ${ }^{1,3,4}$ ASIMINA GKRATSOU, ${ }^{1,2,3}$ CHRISTOPH JOSI, ${ }^{1,2,4}$ MARTINO COLOMBO,,${ }^{1,2}$ \\ and OLIVER MÜHLEMANN ${ }^{1}$ \\ ${ }^{1}$ Department of Chemistry and Biochemistry, University of Bern, Bern 3012, Switzerland \\ ${ }^{2}$ Graduate School for Cellular and Biomedical Sciences, University of Bern, Bern 3012, Switzerland
}

\begin{abstract}
The term "nonsense-mediated mRNA decay" (NMD) originally described the degradation of mRNAs with premature translationtermination codons (PTCs), but its meaning has recently been extended to be a translation-dependent post-transcriptional regulator of gene expression affecting 3\%-10\% of all mRNAs. The degradation of NMD target mRNAs involves both exonucleolytic and endonucleolytic pathways in mammalian cells. While the latter is mediated by the endonuclease SMG6, the former pathway has been reported to require a complex of SMG5-SMG7 or SMG5-PNRC2 binding to UPF1. However, the existence, dominance, and mechanistic details of these exonucleolytic pathways are divisive. Therefore, we have investigated the possible exonucleolytic modes of mRNA decay in NMD by examining the roles of UPF1, SMG5, SMG7, and PNRC2 using a combination of functional assays and interaction mapping. Confirming previous work, we detected an interaction between SMG5 and SMG7 and also a functional need for this complex in NMD. In contrast, we found no evidence for the existence of a physical or functional interaction between SMG5 and PNRC2. Instead, we show that UPF1 interacts with PNRC2 and that it triggers $5^{\prime}-3^{\prime}$ exonucleolytic decay of reporter transcripts in tethering assays. PNRC2 interacts mainly with decapping factors and its knockdown does not affect the RNA levels of NMD reporters. We conclude that PNRC2 is probably an important mRNA decapping factor but that it does not appear to be required for NMD.
\end{abstract}

Keywords: NMD; mRNA turnover; mRNA decapping; UPF1; SMG5; SMG7; PNRC2 tethered function assay

\section{INTRODUCTION}

The nonsense-mediated mRNA decay pathway (NMD) is best known as a post-transcriptional surveillance pathway that specifically identifies and degrades mRNAs containing a premature translation-termination codon (PTC) and hence prevents the build-up of potentially harmful truncated proteins (Lykke-Andersen and Jensen 2015; Hug et al. 2016; Karousis et al. 2016). Messenger RNAs may contain PTCs due to nonsense or frame-shift mutations, transcriptional errors, gene rearrangements, or alternative splicing (Nicholson et al. 2010). In mammalian cells, one-third of alternatively spliced transcripts have PTCs and are substrates for NMD (Lewis et al. 2003). However, in recent years it has been found that NMD controls a larger and more diverse inventory of transcripts than previously thought. Various genome-wide studies in $S$. cerevisiae, D. melanogaster, and human cells showed that $3 \%-10 \%$ of all mRNAs are regulated by the

\footnotetext{
${ }^{3}$ These authors contributed equally to this work.

${ }^{4}$ Present address: Institute of Veterinary Bacteriology, Vetsuisse, University of Bern, Bern 3012, Switzerland

Corresponding author: oliver.muehlemann@dcb.unibe.ch

Article is online at http://www.rnajournal.org/cgi/doi/10.1261/rna. 063719.117. Freely available online through the RNA Open Access option.
}

NMD factor Up-frame shift 1 (UPF1), most of them lacking a PTC (He et al. 2003; Mendell et al. 2004; Rehwinkel et al. 2005; Colombo et al. 2017), which signifies a role for NMD as a translation-dependent post-transcriptional regulator of gene expression.

Past and current research aimed at elucidating the mechanism of NMD led to the structural and functional characterization of many of the factors involved in NMD and to how these factors interact with each other on the target mRNA during the process of NMD (Kervestin and Jacobson 2012; Llorca 2012; Schweingruber et al. 2013). A working model that has emerged from all of this information proposes that the decision of whether NMD is executed or not depends on a kinetic competition between activation of UPF1, a core NMD factor which has $5^{\prime}-3^{\prime}$ helicase and nucleic aciddependent ATPase activities (Bhattacharya et al. 2000), and efficient translation termination and recycling of the ribosomal subunits to the $5^{\prime}$ end of the mRNA in a process that involves cytoplasmic poly(A)-binding protein (PABP), eukaryotic release factor 3 (eRF3), and eukaryotic initiation

(C) 2018 Nicholson et al. This article, published in $R N A$, is available under a Creative Commons License (Attribution-NonCommercial 4.0 International), as described at http://creativecommons.org/licenses/by-nc/4.0/. 
factor 4G (eIF4G) (Czaplinski et al. 1998; Eberle et al. 2008; Ivanov et al. 2008; Silva et al. 2008; Singh et al. 2008; Fatscher et al. 2014; Joncourt et al. 2014). In mammals, degradation of NMD-targeted mRNAs involves both exonucleolytic and endonucleolytic pathways (Schweingruber et al. 2013). The different decay routes all seem to require phosphorylated UPF1 (P-UPF1), which has been shown to interact with the known downstream effectors SMG6, heterodimeric SMG5-SMG7 and Proline-Rich Nuclear Receptor Coactivator 2 (PNRC2) (Cho et al. 2009; Okada-Katsuhata et al. 2012; Jonas et al. 2013). SMG5, SMG6, and SMG7 are related proteins that each contain tetratricopeptide (TPR) domains which form well-conserved phosphoserine binding sites similar to those found in 14-3-3-like proteins (Fukuhara et al. 2005; Jonas et al. 2013). SMG6 and SMG7 have been experimentally confirmed to bind phosphorylated threonine 28 and serine 1096 of UPF1, respectively (Fukuhara et al. 2005; Okada-Katsuhata et al. 2012; Chakrabarti et al. 2014; Nicholson et al. 2014) and SMG5, SMG6, and SMG7 are thought to promote dephosphorylation of UPF1 by recruiting protein phosphatase 2A (Yamashita 2013). The phosphorylation-dephosphorylation cycles are essential for NMD in metazoans and appear to be coordinated with the UPF1 ATPase activity, because ATPase deficient UPF1 accumulates in a hyperphosphorylated form which possibly prevents translation reinitiating on the NMD mRNP (Page et al. 1999; Ohnishi et al. 2003; Kashima et al. 2006; Isken et al. 2008).

SMG6 is a NMD-specific endonuclease that cleaves mRNAs in the vicinity of the PTC using its C-terminal PIN (PilT N terminus) domain (Glavan et al. 2006; Huntzinger et al. 2008; Eberle et al. 2009). SMG6 endonuclease activity depends on SMG1 and UPF1 and requires both the binding of SMG6 to P-UPF1 as well as a phosphorylation-independent interaction between SMG6 and the helicase and SQ domains of UPF1 (Okada-Katsuhata et al. 2012; Chakrabarti et al. 2014; Nicholson et al. 2014).

SMG5 and SMG7 form a heterodimer predominately via their 14-3-3-like domains interacting in an atypical perpendicular back-to-back orientation (Jonas et al. 2013). The ability of this complex to degrade mRNA seems to lie in the C terminus of SMG7, which was shown to recruit the CCR4-NOT deadenylase complex by specifically interacting with the catalytic subunit 8 of the CCR4-NOT transcription complex (CNOT8, also known as POP2) (Loh et al. 2013). Moreover, the tethered C terminus of SMG7 promoted reporter mRNA decay in a decapping enzyme homolog 2 (DCP2) and $5^{\prime}-3^{\prime}$ exoribonuclease 1 (XRN1) dependent manner (Unterholzner and Izaurralde 2004). Thus, it is thought that binding of SMG5-SMG7 to P-UPF1 induces deadenylation-dependent decapping followed by $5^{\prime}-3^{\prime}$ degradation of NMD targets (Unterholzner and Izaurralde 2004; Loh et al. 2013).

Besides binding to SMG7, SMG5 has also been reported to form a complex with PNRC2 and UPF1 to bring about degradation of NMD substrates by virtue of the fact that PNRC2 can bind to decapping mRNA enzyme 1A (DCP1A) (Albers et al. 2005; Cho et al. 2009, 2013; Lai et al. 2012). Specifically, structural and interaction mapping studies have shown that the proline rich region of PNRC2 interacts with the EVH1 domain of DCP1a and that the NR box located in the C terminus of PNRC2 interacts with P-UPF1 (Lai et al. 2012). Accordingly, it has been proposed that P-UPF1 binds SMG5-PNRC2, which in turn recruits DCP1A, and this mediates decapping and presumably $5^{\prime}-3^{\prime}$ degradation of NMD targets. To date, it is not known which phosphorylated residues of UPF1 are involved in the binding of SMG5-PNRC2. Furthermore, it has been reported that this complex is functionally dominant compared to the SMG5-SMG7 in mammalian NMD, since SMG5 was reported to preferentially complex with PNRC2 and UPF1 over SMG7 and UPF1 (Cho et al. 2013). However, this dominance of the PNRC2-SMG5-mediated NMD mode was challenged by reporting the absence of a detectable interaction between PNRC2 and SMG5 (Loh et al. 2013) and thus its functional dominance over the SMG5-SMG7-mediated NMD pathway is unclear and requires further investigation.

In the present study, we have examined the roles of SMG5, SMG7, and PNRC2 with regard to their interacting proteins and their function in degrading mRNA. We could neither detect an interaction between SMG5 and PNRC2 nor find any evidence for SMG5, PNRC2, and UPF1 working together to induce mRNA decay in tethered function assays. Rather, tethered SMG5 predominantly depends on SMG7 to induce mRNA decay, and we clearly observed an interaction between these two proteins. Moreover, we confirmed the reported interaction between UPF1 and PNRC2 and provide evidence that tethered UPF1 requires PNRC2 to induce decapping followed by $5^{\prime}-3^{\prime}$ exonucleolytic mRNA decay. However, this pathway does not appear to be required for NMD in vivo because NMD reporter transcript levels were unaffected in PNRC2 knockdown cells. Collectively, our data suggest that PNRC2 is a constituent of the general decapping complex that does not seem to have a specific function in NMD, while UPF1 seems to be an important universal mRNA degradation factor that is essential for NMD, but not exclusive to NMD. Therefore, our data emphasize the need for a commonly agreed upon definition of NMD, which ultimately may turn out to be a combination of several different, yet overlapping mechanisms that all result in reduced levels of a specific subset of mRNAs.

\section{RESULTS}

\section{Tethering of SMG5, SMG7, PNRC2, and UPF1 all induce a strong reduction of reporter $\mathrm{mRNA}$}

To further our understanding of the role of UPF1, SMG5, SMG7, and PNRC2 in the degradation of NMD substrates, we set up a tethered function assay (TFA) as shown in Figure 1A (see Nicholson et al. 2012 for a detailed 
A

Tethered Function Assay

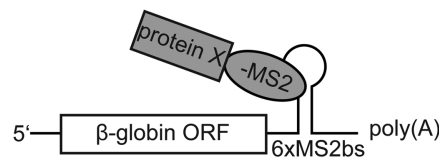

B

C

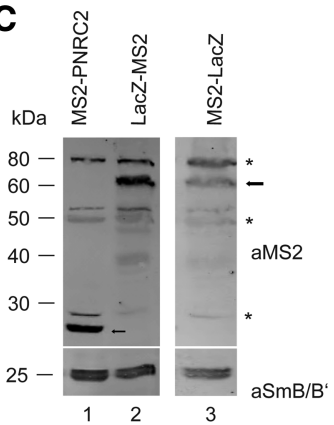

FIGURE 1. Tethered SMG5, SMG7, PNRC2, and UPF1 all strongly reduce reporter mRNA levels bypassing the need for a PTC. (A) Schematic of the MS2-tethered function assay (TFA), where protein X represents UPF1, SMG5, SMG7, or PNRC2. (B) TFA results. Relative $\beta$-globin reporter mRNA levels, normalized to EGFP mRNA levels and with reporter mRNA levels in cells expressing LacZ-MS2 set as 100, were determined by RT-qPCR from total RNA of HeLa cells transfected with pc $\beta$-globin $6 \mathrm{xMS} 2 \mathrm{bs}$, pEGFP (serving as a cotransfection control) and with plasmids expressing each of the indicated proteins fused to a MS2 moiety. The mean and standard deviations (SDs) of $>4$ independent experiments are indicated, wherein $\left({ }^{* *}\right)$ denotes $P \leq 0.01$ as determined by Student's $t$-tests. $(C)$ Western blots showing the expression of MS2-PNRC2, LacZ-MS2, and MS2-LacZ fusion proteins (left panel), the MS2-UPF1 fusion protein (middle panel), and the MS2-SMG5 and MS2-SMG7 fusion proteins (right panel). The antibodies used are indicated at the right of each blot. Arrows denote the correct bands while $\left(^{*}\right)$ indicates unspecific bands detected with the anti-MS2 antibody. Actin or $\mathrm{SmB} / \mathrm{B}^{\prime}$ served as loading controls in the western blots.

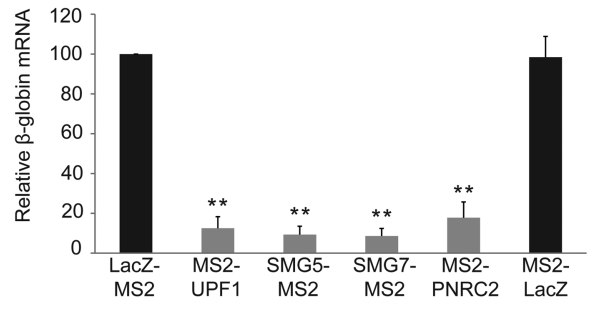

teins were tethered to a Renilla luciferase reporter mRNA containing six MS2 binding sites in its $3^{\prime}$ UTR (Supplemental Fig. S1). Thus, UPF1, SMG5, SMG7, and PNRC2 can all cause a strong reduction in reporter mRNA levels when tethered to the 3'-UTR. Western blots were performed to document the expression of the MS2-fusion proteins in the TFA (Fig. 1C).

\section{SMG5-mediated reporter mRNA level reduction requires $\mathrm{SMG7}$}

To examine which other factors were required for the strong reporter level reductions induced by the tethered SMG5 in Figure 1B, we performed TFAs with SMG5-MS2 in cells wherein UPF1, SMG1, SMG6, SMG7, or PNRC2 levels were reduced by expressing the corresponding shRNAs, while expression of a shRNA with no predicted target RNA (Ctr KD) served as a control. Part of the cell lysates were used to extract RNA and determine the relative $\beta$-globin reporter mRNA levels (Fig. 2A), while western blots were performed with the remainder of each lysate to assess the knockdown efficiencies of the stipulated factors (Fig. 2B).

description). Full-length SMG5, SMG7, PNRC2, and UPF1, and a fragment of LacZ serving as a control, were fused to the MS2 coat protein. The indicated MS2-fusion proteins were coexpressed with a $\beta$-globin reporter mRNA containing six MS2 binding sites in its $3^{\prime}$ UTR ( $\beta$-globin-6xMS2bs) and a GFP expressing plasmid in HeLa cells, and the steady-state levels of the reporter mRNA were quantified and normalized to the levels of GFP mRNA (Fig. 1B; Supplemental Table S1 for $P$-values). Expression of SMG5 and SMG7-MS2-fusion proteins strongly reduced the steady-state levels of the reporter mRNA levels to below $10 \%$ compared to cells expressing the LacZ-MS2 or the MS2-LacZ controls, which encode a fragment of LacZ fused to a C-terminally or an $\mathrm{N}$-terminally located MS2 moiety, respectively. Similarly, expression of MS2-UPF1 or MS2-PNRC2 fusion proteins also strongly reduced the reporter mRNA levels to $12 \%$ and $18 \%$, respectively. These results confirm previous TFAs conducted with UPF1 (Lykke-Andersen et al. 2000; Chiu et al. 2003; Cho et al. 2009, 2013), PNRC2 (Cho et al. 2009, 2013) and SMG7 (Unterholzner and Izaurralde 2004; Cho et al. 2013; Jonas et al. 2013; Loh et al. 2013). Reduced reporter mRNA levels, albeit to a lesser extent than observed here, were also reported by others with SMG5-MS2 (Cho et al. 2013; Loh et al. 2013). The same results were obtained when the MS2-fusion pro-
Since we were not able to detect neither endogenous PNRC2 nor overexpressed myc-tagged or MS2-tagged PNRC2 with any of the tested anti-PNRC2 antibodies (see Supplemental Fig. S2), we documented the effectiveness of all of the designated RNAi-mediated knockdowns also at the mRNA level (Supplemental Fig. S3). Expression of PNRC2 mRNA-targeting shRNA resulted in a strong reduction of endogenous PNRC2 mRNA and efficient depletion of exogenously expressed MS2-PNRC2 fusion protein, documenting the effectiveness of our PNRC2 knockdown (Fig. $2 \mathrm{~B}$, compare lanes 1 and 3 to 2 and 4 ).

The $\beta$-globin reporter mRNA level for each experimental condition is depicted relative to the level in cells expressing LacZ-MS2 and normalized to GFP mRNA expressed from a cotransfected plasmid to account for possible differences in transfection efficiencies among the samples (Fig. 2A; Supplemental Table S2 for $P$-values). The ability of tethered SMG5-MS2 to down-regulate the reporter mRNA predominately requires SMG7 because the SMG7 knockdown compromised the extent of reporter mRNA reduction by fivefold. In contrast, knockdown of UPF1 or SMG6 did not affect the activity of SMG5-MS2, while knockdown of SMG1 or PNRC2 very slightly compromised the SMG5MS2-mediated reporter mRNA decay (Fig. 2A). 

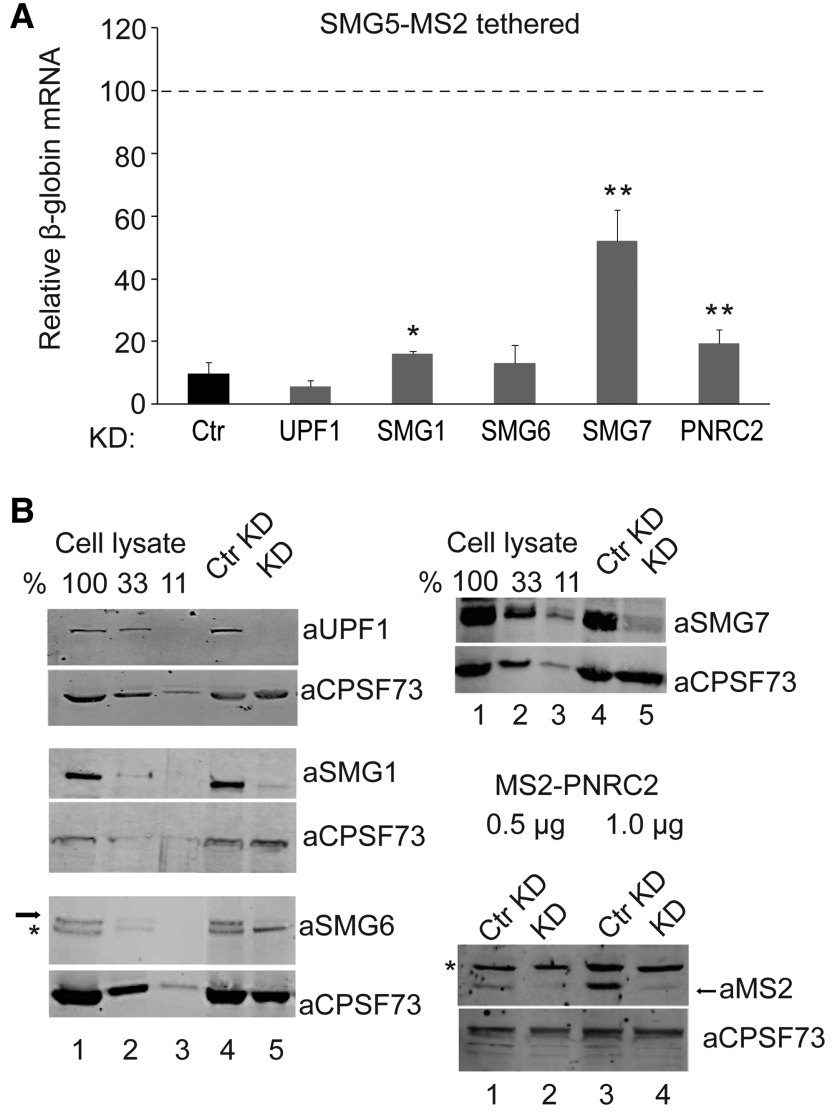

FIGURE 2. SMG5-mediated reporter mRNA decay requires SMG7. (A) TFA of SMG5-MS2 as in Figure 1, except performed in cells with a knockdown (KD) of the factors indicated at the bottom of each panel. Ctr denotes cells expressing a control shRNA. Relative $\beta$-globin reporter mRNA levels were normalized to EGFP mRNA levels and with mRNA levels in cells expressing LacZ-MS2 set as 100 (dashed line). The Ctr knockdown condition (black bar) serves as reference to assess the effects caused by depletion of the indicated factors in the respective tethering assays. The mean and SD of $>4$ independent experiments are shown. $P$-values $\leq 0.01$ are indicated as $\left({ }^{* *}\right)$ and $\leq 0.05$ as $\left({ }^{*}\right)$. $(B)$ Western blots examining the knockdown efficiencies of the NMD factors from Figure 2A. Lanes $1-3$ show serial dilutions (100\%, 33\%, and $11 \%$ ) of cell lysate from untransfected HeLa cells (Plain cells). Lanes 4 and 5 display the protein levels from cells expressing a control shRNA (Ctr KD) and the shRNA targeting the designated NMD factor (KD), respectively. The antibodies used are indicated at the right of each blot. The efficiency of the PNRC2 knockdown assessed using an anti-MS2 antibody is documented using cells transfected with plasmids expressing either a control shRNA (Ctr KD; lanes 1 and 3) or shRNAs targeting PNRC2 (KD; lanes 2 and 4), along with the indicated amount of plasmid expressing MS2-PNRC2. Arrows represent the correct bands while the $\left({ }^{*}\right)$ points to unspecific bands. Detection of CPSF-73 levels served as a loading control.

Since it was shown that the SMG5-SMG7 heterodimer recruits the CCR4-NOT deadenylase complex via the C-terminal half of SMG7 (Loh et al. 2013), we predicted that in the TFA, SMG5-induced reporter RNA degradation would depend on this so-called PC region of SMG7 (Fig. 3A; Unterholzner and Izaurralde 2004). To test this, we coexpressed the $\beta$-globin-6xMS2bs reporter RNA, SMG5-MS2, and RNAi-resistant versions of SMG7 $\left(\mathrm{WT}^{\mathrm{R}}, \Delta \mathrm{C}^{\mathrm{R}}, \Delta \mathrm{PC}^{\mathrm{R}}\right.$ ) in cells depleted of endogenous SMG7. As before, part of the cell lysates was used to extract RNA and determine the relative $\beta$-globin reporter mRNA levels (Fig. 3B; Supplemental Table S3 for $P$-values) and the remainder of each lysate was used for western blotting to assess the knockdown efficiency of endogenous SMG7 and the expression levels of the RNAi-resistant SMG7 constructs (Fig. 3C). The finding that SMG7-WT ${ }^{\mathrm{R}}$ rescued the ability of tethered SMG5 to decrease the reporter RNA levels to a similar extent as observed in the control knockdown (Ctr) was expected (Fig. 3B). However, we were initially surprised to observe that the SMG7 constructs lacking the C-terminal amino acids 838-1091 $\left(\Delta \mathrm{C}^{\mathrm{R}}\right)$ and even 633-1091 $\left(\Delta \mathrm{PC}^{\mathrm{R}}\right)$ also rescued tethered SMG5 activity, since they cannot interact with the CCR4-NOT complex anymore (Loh et al. 2013).

We speculated that tethered SMG5 to some extent might also down-regulate the reporter RNA through its 14-3-3 domain interacting with SMG7 and as a heterodimer recruiting UPF1. Therefore, we repeated the SMG5 TFA/SMG7 rescue experiment in cells depleted for both, SMG7 and UPF1 (Fig. 3D,E; Supplemental Table S3 for $P$-values). Compared to the control knockdowns expressing a scrambled shRNA (lanes 1,2), UPF1 and SMG7 protein levels were reduced to $<20 \%$ and below the level of detection, respectively (lanes 3-6, Fig. 3E). This codepletion of UPF1 and SMG7 reduced the down-regulation of the reporter RNA in response to SMG5 tethering by 4.5 -fold from $14 \%$ to $63 \%$ (Fig. 3D, compare Ctr to dKD). Expression of RNAi-resistant wildtype SMG7 completely rescued the SMG5-induced reporter RNA down-regulation $\left(\mathrm{dKD} \mathrm{WT}^{\mathrm{R}}\right)$, whereas the rescuing capacity of SMG7- $\Delta C^{\mathrm{R}}$, although expressed to higher levels than SMG7-WT ${ }^{\mathrm{R}}$, was partially compromised $\left(\mathrm{dKD} \Delta \mathrm{C}^{\mathrm{R}}\right)$ and the SMG7 lacking the entire C-terminal half did not rescue at all $\left(\mathrm{dKD} \Delta \mathrm{PC}^{\mathrm{R}}\right)$.

Collectively, our results demonstrate that in TFA, SMG5 induces the degradation of the reporter RNA primarily through its interaction with SMG7 and that this RNA decay pathway depends on the C-terminal PC region of SMG7, in agreement with a previous study showing that this region recruits the CCR4-NOT deadenylase complex (Loh et al. 2013).

\section{Yeast two-hybrid assays confirm SMG5-SMG7 and UPF1-PNRC2 interactions but do not identify a SMG5-PNRC2 interaction}

Given our results above and the partial inconsistencies with other studies (Cho et al. 2013), we decided to examine the protein-protein interaction networks between UPF1, SMG5, SMG7, and PNRC2 using yeast two-hybrid assays (Fields and Song 1989). First, we tested the interaction of SMG7 fused to the GAL4-activation domain (-AD) against UPF1, PNRC2, and SMG5, all fused to the GAL4-DNA-binding domain (-BD) (Fig. 4A). Usage of the $L a c Z$ reporter gene allowed detection of colonies expressing $\beta$-galactosidase by addition 


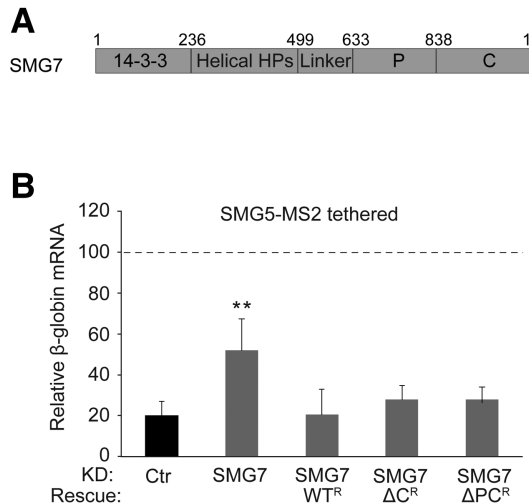

D

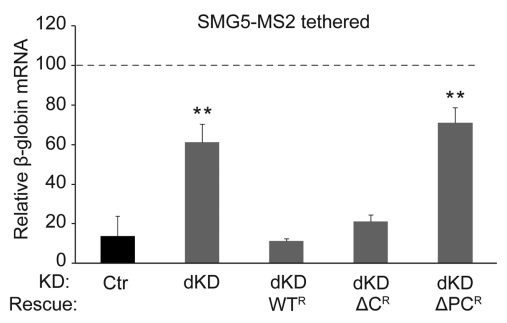

C

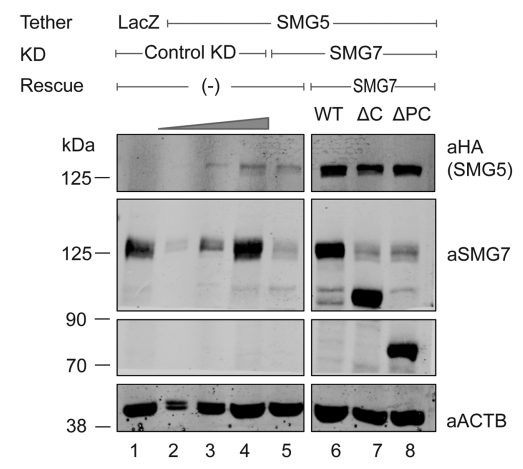

E

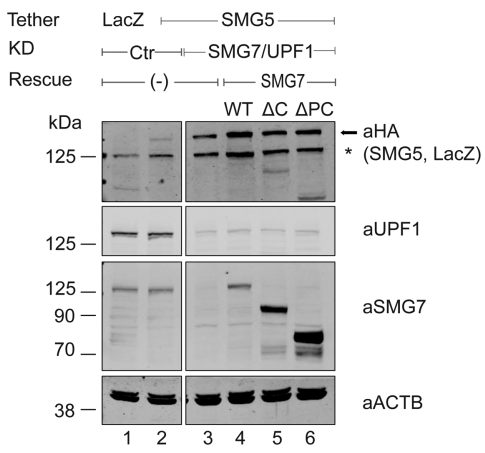

FIGURE 3. SMG5-MS2 requires the SMG7 PC region for reporter mRNA degradation. (A) The SMG7 domain architecture is schematically depicted (Unterholzner and Izaurralde 2004). (B,D) SMG5-MS2 TFAs, performed in cells with SMG7 knockdown in $B$ or with a double SMG7/UPF1 knockdown (dKD) in $D$ and rescuing with the indicated RNAi-resistant SMG7 constructs $\left(\mathrm{WT}^{\mathrm{R}}\right.$, wild-type; $\Delta \mathrm{C}^{\mathrm{R}}$, lacking the $\mathrm{C}$ terminus; $\Delta \mathrm{PC}^{\mathrm{R}}$, lacking the whole $\mathrm{PC}$ region). Relative $\beta$-globin reporter mRNA levels were normalized to EGFP mRNA levels and with mRNA levels in cells expressing LacZ-MS2 set as 100. The control knockdown conditions (black bars) serve as reference to assess the effects of the tethered protein, the depletion of the indicated factors and the rescue with the corresponding constructs. The mean and SD of $>4$ independent experiments are shown, with $P$-values $\leq 0.01$ depicted as $\left({ }^{* *}\right)$. $(C, E)$ Western blots examining the knockdown efficiencies of the NMD factors and the protein levels of the SMG7 rescue constructs from $B$ and $D$, respectively. The antibodies used are indicated at the right of each blot. $(C)$ Lanes 2-4 depict serial dilutions $(11 \%, 33 \%$, and $100 \%)$ of the control knockdown sample. (E) The unspecific band detected with the anti-HA antibody is denoted with a $\left({ }^{*}\right)$, and the bands corresponding to HA-SMG5 and HA-LacZ are indicated by arrows. Detection of Actin levels served as a loading control.

of the chromogenic substrate X-gal to the plates. Of each cotransformation, four colonies (A-D) were analyzed. Coexpression of BD-UPF1 and AD-SMG7 resulted in weak blue colonies (Fig. 4A, row 2), indicating a weak interaction between UPF1 and SMG7. While we did not observe an interaction between BD-PNRC2 and AD-SMG7 (row 3), we scored a strong blue color and hence interaction between the BDSMG5 and AD-SMG7 (row 4). While the observed strong interaction between SMG5 and SMG7 is in line with previous reports (Anders et al. 2003; Ohnishi et al. 2003; Jonas et al. 2013) we could not observe the recently documented SMG5PNRC2 interaction (Cho et al. 2013) in our yeast two-hybrid assays (row 12). However, coexpression of BD-UPF1 and ADPNRC2 resulted in blue colonies (row 6), signifying an interaction between the UPF1 and PNRC2. Furthermore, by expressing UPF1 deletion mutants fused to the $\mathrm{BD}$, we were

able to decipher that PNRC2 interacts with the N-terminal first 271 amino acids of UPF1, which comprises the cysteinehistidine rich $(\mathrm{CH})$ domain, and not with the helicase domain (HD) or the C-terminal serine-glutamate rich (SQ) domain (Fig. 4A, compare rows 6-11). The BD-only controls showed that none of the proteins fused to the -AD were self-activating in these assays (rows 1 and 5), and the AD-only controls confirmed that none of the proteins fused to the $\mathrm{BD}$ were able to cause self-activation (rows 13-16). BD-eRF3a and AD-eRF1 were included as a positive control (row 17), since the interaction between eRF1 and eRF3 is well established (Stansfield et al. 1995; Zhouravleva et al. 1995). We also performed the same assays with PNRC2 and SMG7 fused to the DNABD and SMG5 and UPF1 fused to the $\mathrm{AD}$ and the reciprocal fusions for eRF1 and eRF3. By performing the assays with the proteins fused to the opposite part of the GAL4 transcription factor, we found that SMG7 fused to the BD causes strong self-activation (data not shown). We could not detect an interaction between BD-SMG5 and AD-UPF1 or between AD-SMG5 and BD-PNRC2 but we could confirm an interaction between ADUPF1 and BD-PNRC2 (Supplemental Fig. S4A,B).

In addition to the directed yeast two-hybrid assays described above, we also carried out a small yeast mating screen (Finley and Brent 1994) to examine the various interactions between UPF1, SMG5, SMG7, PNRC2, and other NMD factors (Supplemental Fig. S5A,B). One mating type (MATa) expressing proteins fused to the $\mathrm{AD}$ was mated with the opposite mating type (MATa) expressing proteins fused to the BD to generate diploids that contained both constructs and were subsequently tested for protein-protein interactions by assaying the ADE2 and the HIS3 reporter genes. The former was done by testing for growth on medium lacking adenine and the latter was performed by testing for growth on medium lacking histidine but containing 2.5 mM 3-AT. Supplemental Figure S5 shows the results of screens carried out using at least ten different transformants of the designated proteins fused to the AD. Two reporter genes were assayed for activation and all the interactions found in $\geq 80 \%$ or $\geq 60 \%$ are indicated by dark green or light green colored boxes, respectively. The yeast mating screen substantiated our findings from the directed yeast two-hybrid 
A

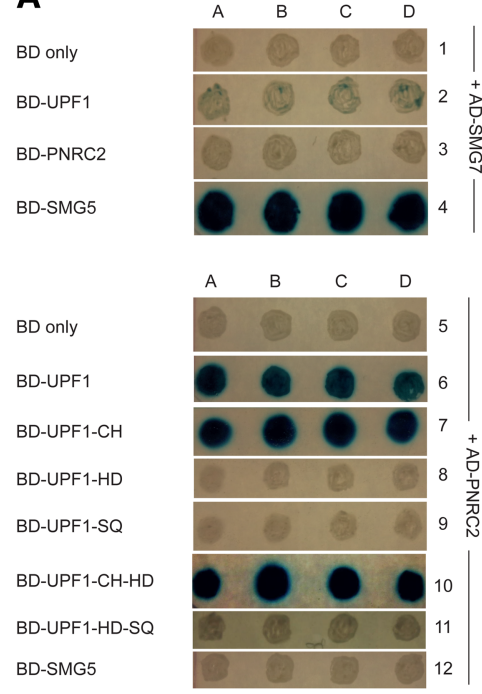

B

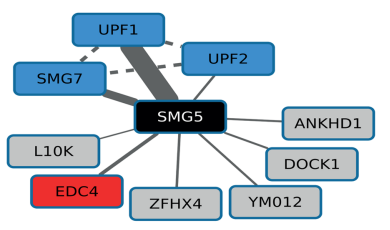

C

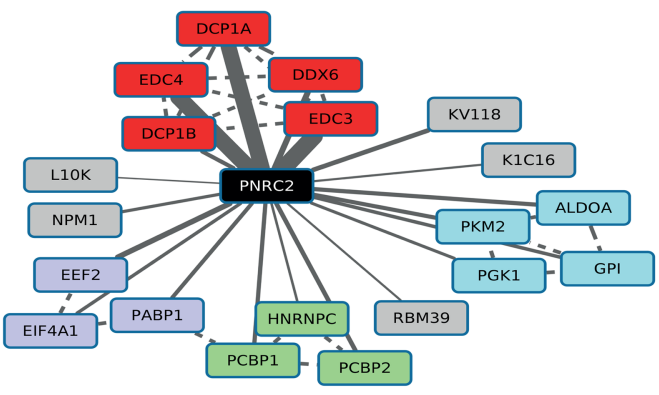

FIGURE 4. PNRC2 interacts with decapping complex factors and UPF1 but not with SMG5. (A) Yeast two-hybrid assay. Plasmids expressing the indicated protein variants fused to the GAL4DNA-binding domain (BD-) were cotransformed with plasmids expressing the GAL4-activation domain fused to SMG7 (AD-SMG7) or PNRC2 (AD-PNRC2) into Mav99 cells. The UPF1 constructs that were used for mapping the UPF1-PNRC2 interaction are depicted (see schematic). Four colonies (denoted A-D) from each cotransformation were selected for the $\beta$-galactosidase assay. $\mathrm{BD}$ only denotes expression of the $\mathrm{BD}$ alone and served to control for self-activation of the cotransformed $\mathrm{AD}$-construct (rows 1, 5, and 13). $\mathrm{AD}$ only represents transformation of a plasmid expressing GAL4-AD-empty. The known interaction between BD-eRF3 and AD-eRF1 served as a positive control (row 17). (B,C) Schematic representation of the top ranked proteins identified by shotgun LC-MS/MS in the immunoprecipitates of SMG5-MS2 (B) or of MS2-PNRC2 $(C)$. The diagrams were produced with Cytoscape (v3.1.1). Every protein present in the mass spectrometry data is reported as a node that is connected with an edge to the protein used for the immunoprecipiation (displayed as the black central node). The width of this edge is proportional to the square root of the protein match score summation (PMSS) of the respective protein. The dashed lines between proteins indicate already documented protein-protein interactions according to the STRING data set (v9.1) with a threshold of the top 20 high confidence interactors of every protein. Every node connected by at least one dashed line was colored in the same way and the legend is shown in the upper right corner. The list of proteins identified to be copurifying with MS2-LacZ, SMG5-MS2, and MS2-PNRC2 are listed in Supplemental Tables S4-S6, respectively. assays in that we found interactions between AD-UPF1 and BD-PNRC2, as well as between AD-PNRC2 and BDUPF1, and we could again specifically map this interaction point to the $\mathrm{N}$-terminal 271 amino acids of UPF1 (Supplemental Fig. S5A). Furthermore, we found that AD-PNRC2 interacted with BDPNRC2, opening up the possibility that PNRC2 may function as a dimer. Again, we could not identify any interaction between AD-PNRC2 and BD-SMG5, nor could we score an interaction between AD-SMG5 and BD-PNRC2. In contrast to the directed yeast two-hybrid assays, we detected in the mating assay the previously reported interaction between UPF1 and SMG5 with both AD-UPF1 and BDSMG5 as well as with AD-SMG5 and BDUPF1 but could not map the SMG5 interaction to a specific part of UPF1, suggesting that SMG5 may interact with various domains of UPF1. For the mating assays, we always fused the desired proteins to both the DNA-BD and $\mathrm{AD}$, since this allows the possibility to escape any steric hindrances, misfolding or low expression that may occur in one particular protein fusion constellation. Due to this, we could identify protein fusions that caused reporter gene self-activation and this is the reason why the screens are not identically reciprocal.

As described above and confirming all of our earlier findings, we observed again PNRC2 interacting with itself and with UPF1, but not with SMG5, while we scored an interaction between BDSMG5 and AD-SMG7 (Supplemental Fig. S5B). Thus, the lack of detecting an interaction between SMG5 and PNRC2 cannot be due to one or both fusion proteins not expressing in the yeast cells. We could also identify previously characterized UPF1 interaction partners such as UPF2 and SMG6 (Clerici et al. 2009; Okada-Katsuhata et al. 2012; Chakrabarti et al. 2014; Nicholson et al. 2014), as well as interactions between UPF1 and SMG7 and UPF1-N and SMG5, albeit with lower confidence.

Four conclusions can be drawn from all of the conducted yeast two-hybrid assays. First, the well-known interaction between human SMG5 and SMG7 could be 
identified for the first time by yeast two-hybrid assays. Second, we conclude that the N-terminal 271 amino acids of UPF1 are sufficient for its interaction with PNRC2, a region that has also been implicated in PNRC2 interaction by coimmunoprecipitation assays (IPs) (Loh et al. 2013). Third, we found that PNRC2 can bind to itself. Finally, our yeast two-hybrid assays did not provide any evidence for an interaction between SMG5 and PNRC2. In addition, we see evidence for potential phosphorylation-independent interactions between SMG7, PNRC2 and perhaps also SMG5 with UPF1, since UPF1 phosphorylation most likely does not occur in yeast cells as they lack a SMG1 ortholog (Yamashita 2013). The possibility of SMG5 and SMG7 being able to bind UPF1 in a phosphorylation-independent manner has been previously hinted at (Ohnishi et al. 2003; Okada-Katsuhata et al. 2012; Jonas et al. 2013) and such an interaction has been shown for SMG6 and UPF1 to be crucial for NMD (Chakrabarti et al. 2014; Nicholson et al. 2014).

\section{PNRC2 interacts with decapping factors while SMG5 interacts with NMD factors}

To further search for the previously reported interaction between PNRC2 and SMG5 (Cho et al. 2013) that we could not detect in our yeast two-hybrid assays, we used a complementary approach where we immunoprecipitated MS2SMG5, MS2-PNRC2 and MS2-LacZ (the latter serving as a control) from RNase A-treated cell lysates followed by shotgun liquid chromatography coupled to two stage mass spectrometry (Shotgun LC-MS/MS). The immunoprecipitated proteins were digested on the beads with trypsin and the resulting peptide mixture was directly analysed by LC-MS/ MS. Proteins that were also found copurifying with our control MS2-LacZ (Supplemental Table S4) were discarded and an overview of the remaining proteins identified in the MS2-SMG5 and MS2-PNRC2 IP samples are shown in Supplemental Tables S5 and S6, respectively. We have diagrammatically represented in Figure $4 \mathrm{~B}$ and $\mathrm{C}$ the top nine and top 20 proteins that were found copurifying with SMG5-MS2 or MS2-PNRC2, respectively, based on the calculated protein match score summation (PMSS).

We found the NMD factors UPF1, SMG7 and UPF2 copurifying with SMG5 and notably we did not find PNRC2 (Fig. 4B). Enhancer of mRNA decapping 4 (EDC4, also known as HEDLS or Ge-1) was the only mRNA degradation factor copurifying with SMG5. Similarly, we did not find SMG5 copurifying with MS2-PNRC2 (Fig. 4C). In fact, we could not identify any bona fide NMD factors copurifying with PNRC2. Instead, the top interactors with PNRC2 were decapping mRNA enzyme 1A (DCP1A), DCP1B, DEAD (Asp-Glu-Ala-Asp) box helicase 6 (DDX6, also known as Rck/p54), enhancer of mRNA decapping 3 (EDC3) and EDC4. Thus, we identified five well-characterized mRNA decapping complex constituents (Houseley and Tollervey 2009; Schoenberg and Maquat 2012) copurifying with
PNRC2 which are known to also interact with each other (indicated by the dashed gray line) (Ling et al. 2011). We also found PNRC2 copurifying various important mRNA translation factors such as poly(A)-binding protein 1 (PABP1), eukaryotic translation initiation factor 4A1 (EIF4A1) and eukaryotic translation elongation factor 2 (EEF2). The interaction of PNRC2 with various mRNA translation factors is not surprising considering that PNRC2 seems to be an mRNA decapping factor and mRNA translation and degradation are closely coupled (Houseley and Tollervey 2009; Schoenberg and Maquat 2012). We also found PNRC2 copurifying with four key glycolytic enzymes which all seem to work closely together, namely the muscle-specific pyruvate kinase 2 (PKM2), fructose-bisphosphate aldolase A (ALDOA), phosphoglycerate kinase 1 (PGK1), and glucose-6-phosphate isomerase (GPI). This is interesting, since it has been reported that PNRC2 may play a role in controlling the balance between energy storage and expenditure (Zhou et al. 2008; Lai et al. 2012). Another interesting group of proteins that copurified with PNRC2 is the heterogeneous nuclear ribonucleoproteins (hnRNPs). Specifically, PNRC2 copurified hnRNP C, hnRNP E1 (also known as PCBP1), and hnRNP E2 (also known as PCBP2).

Thus, we can summarize that in these co-IP experiments, SMG5 primarily interacts with SMG7 and factors known to be involved in the process of NMD, while PNRC2 chiefly interacts with components of the mRNA decapping process, an interaction that has been well documented previously (Lai et al. 2012; Mugridge et al. 2016). Consistently, pathway analysis using the interactome analysis tool yielded NMD and 5'$3^{\prime}$ exoribonucleolytic decay as the top ranked pathway for the SMG5 and PNRC2 data sets, respectively.

\section{PNRC2 coimmunoprecipitates primarily phosphorylated UPF1}

To gain additional information about the interactions among the decapping complex factors DCP1A, DCP2, and PNRC2, and of UPF1 and XRN1 with these decapping factors, we transiently expressed pairs of tagged proteins in HEK293T cells, immunoprecipitated one of the proteins by their respective tag and checked for the copurification of the other protein by western blotting. A first series of IPs was performed with anti-MS2 antibody pulling down MS2-HALacZ, MS2-UPF1, and MS2-PNRC2 (Fig. 5A-C) and in a second series we used anti-GFP and anti-FLAG antibodies to pull down HA-EGFP, FLAG-GAPDH, EGFP-XRN1, FLAGDCP1A and FLAG-DCP2 (Fig. 5D,E). MS2-HA-LacZ, HAGFP and FLAG-GAPDH were used as negative controls, because they are not expected to interact with any of the tested proteins.

As expected, no detectable amounts of EGFP-XRN1, FLAG-DCP1A and FLAG-DCP2 copurified with MS2-HALacZ from HEK293T cell lysates that were not treated with RNase A (Fig. 5A, lanes 5-7). For MS2-UPF1, a very weak 
A
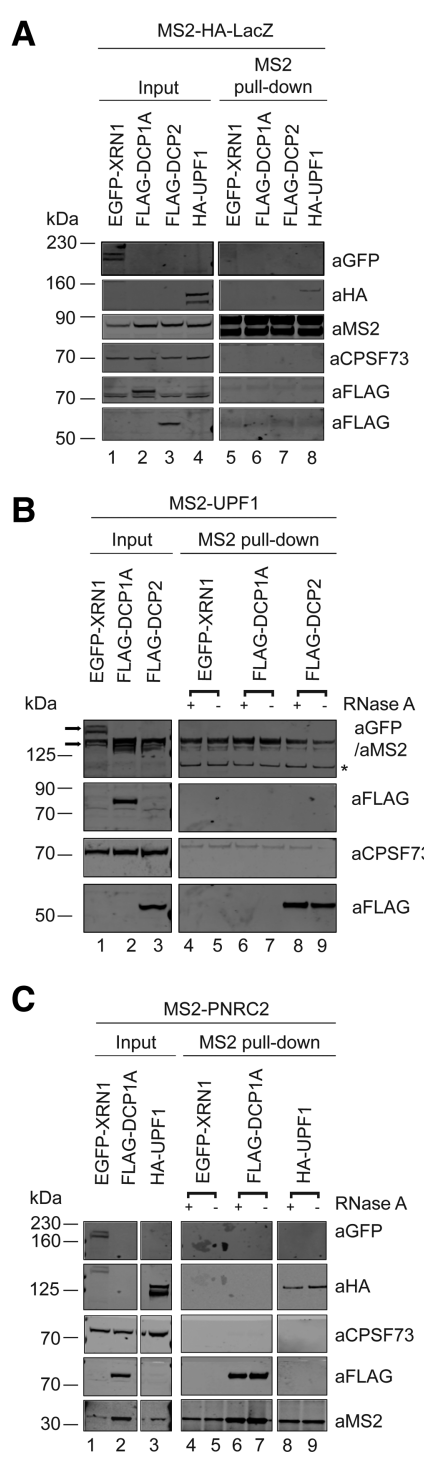

FIGURE 5. UPF1 interacts with PNRC2, decapping complex factors, and XRN1, while PNRC2 coimmunoprecipitates primarily phosphorylated UPF1. (A-E) HEK293T cells were transfected with plasmids expressing pairs of tagged proteins. Three percent of the input material and $100 \%$ of the immunoprecipitated material were loaded on to a $4 \%-12 \%$ gradient gel and analyzed by SDS-PAGE. (A) As a negative control for purifying, the MS2 fusion protein MS2-HA-LacZ was immunoprecipitated from lysates of HEK293T cells coexpressing either EGFP-XRN1, FLAGDCP1A, FLAG-DCP2, or HA-UPF1 using an antibody against the MS2 coat protein. Lanes 1-4 represent the input material and lanes 5-8 show the immunoprecipitated material. $(B)$ Lysates from HEK293T cells coexpressing EGFP-XRN1, FLAG-DCP1A, or FLAG-DCP2 with MS2UPF1 and treated with RNase A $(+)$ or untreated $(-)$ were immunopurified using anti-MS2 antibody. Lanes 1-3 represent the input material and lanes 4-9 show the immunoprecipitated material. $(C)$ Immunoprecipitations performed as in $B$ from lysates of HEK293T cells expressing MS2-PNRC2 and EGFP-XRN1, FLAG-DCP1A, or HA-UPF1. (D) As a negative control for purifying the GFP and FLAG fusion proteins, HA-EGFP and FLAG-GAPDH were purified from HEK293T lysates cotransfected either with MS2-UPF1 or MS2-PNRC2 and using anti-GFP and anti-FLAG antibodies, respectively. Lanes $1-4$ show the input material, while lanes 5-8 represent the immunoprecipitated material. (E) GFP pull-downs from HEK293T lysates expressing EGFP-XRN1 and MS2-UPF1 or MS2-PNRC2 (left panel), FLAG pull-downs from HEK293T lysates expressing FLAG-DCP1A and MS2-UPF1 or MS2-PNRC2 (middle panel), or FLAG-DCP2 and MS2-UPF1 or MS2-PNRC2 (right panel), either treated with RNase A $(+)$ or untreated $(-)$. Lanes $1-2,7-8$, and $13-14$ show the input material, while lanes 3-6, 9-12, and 15-18 show the immunoprecipitated material. CPSF73 served as a loading control for the input material and as a negative control for the purifications. Arrows indicate the correct bands while the $\left(^{*}\right)$ points to an unspecific band detected with the anti-MS2 antibody. band that might correspond to P-UPF1, which is the upper band of the two bands observed in western blots with UPF1 (Flury et al. 2014), was detected in the MS2-HA-LacZ pull-down (lane 8). Endogenous CPSF73 served as a loading control in the input samples and as an additional specificity control for the IPs. Under the same conditions, FLAGDCP2, but no detectable amounts of EGFP-XRN1 and FLAG-DCP1A copurified with MS2-UPF1 (Fig. 5B). The interaction between UPF1 and DCP2 resisted RNase A treatment of the lysate, indicating that it is not bridged by RNA but rather protein-protein mediated (Fig. $5 \mathrm{~B}$, compare lanes 8 and 9). MS2PNRC2 copurified DCP1A and the phosphorylated form of UPF1 but no detectable amounts of EGFP-XRN1 (Fig. 5C). The PNRC2 interactions with both, DCP1A and P-UPF1, were RNase A resistant (Fig. 5C, compare lanes 6 with 7 and 8 with 9). Since the proportion of copurified P-UPF1 was much higher with MS2PNRC2 than with MS2-HA-LacZ, in particular when taking into account the much more efficient pull-down of MS2HA-LacZ compared to MS2-PNRC2, this data suggests that PNRC2 preferentially interacts with P-UPF1.

In the second series of IPs, the control HA-EGFP did not copurify any MS2UPF1 or MS2-PNRC2 (Fig. 5D, lanes 5 and 6). The control FLAG-GAPDH also did not copurify any MS2-PNRC2 (lane 8 ), while trace amounts of MS2-UPF1 were detected (lane 7), suggesting that UPF1 has a slight tendency to co-IP promiscuously. These control IPs were performed without RNase A treatment of the lysates to allow for the detection of both, RNA-mediated and RNA-independent interactions. Much higher amounts of MS2-UPF1 than observed with the controls copurified with EGFP-XRN1, FLAG-DCP1A and FLAG-DCP2 in an RNA-independent manner (Fig. 5E, lanes 3, 4, 9, 10, 15, and 16). Notably, FLAG-DCP1A appeared to preferentially co-IP P-UPF1 (i.e., the uppermost of the bands visible in the input, compare lane 7 with lanes 9 and 10). Substantial amounts of MS2-PNRC2 copurified with FLAG-DCP1A regardless of RNase 
A treatment (lanes 11 and 12), as demonstrated previously (Lai et al. 2012), while the EGFP-XRN1 pull-down copurified only trace amounts (lanes 5 and 6) and FLAG-DCP2 did not bring down any detectable amounts of MS2-PNRC2 (lanes 17 and 18). However, it should be noted that the failure to detect PNRC2 in the DCP2 IP may be due to too little starting material, since the lysate for this IP appeared to contain very low amounts of FLAG-DCP2 (lane 14). Unfortunately, low FLAG-DCP2 expression was a recurrent issue in these experiments.

Collectively, these IP results are in good agreement with our yeast two-hybrid data (Fig. 4A), our mass spectrometry data (Fig. 4C) and previous studies (LykkeAndersen 2002; Cho et al. 2009; Lai et al. 2012; Loh et al. 2013) by confirming the interactions between preferentially phosphorylated UPF1 and PNRC2, between PNRC2 and DCP1A, and between DCP2 and UPF1 independent of UPF1's phosphorylation status. Furthermore, we provide evidence for an interaction between UPF1 and XRN1, even though it was only detected when pulling at EGFP-XRN1 but not when pulling at MS2-UPF1. Altogether, the results indicate that P-UPF1 may recruit the decapping complex to mRNA via contacts with PNRC2 and DCP2.

\section{Tethered UPF1 reduces reporter mRNA levels through PNRC2 and XRN1 but independently of the NMD effectors SMG5, SMG6, and SMG7}
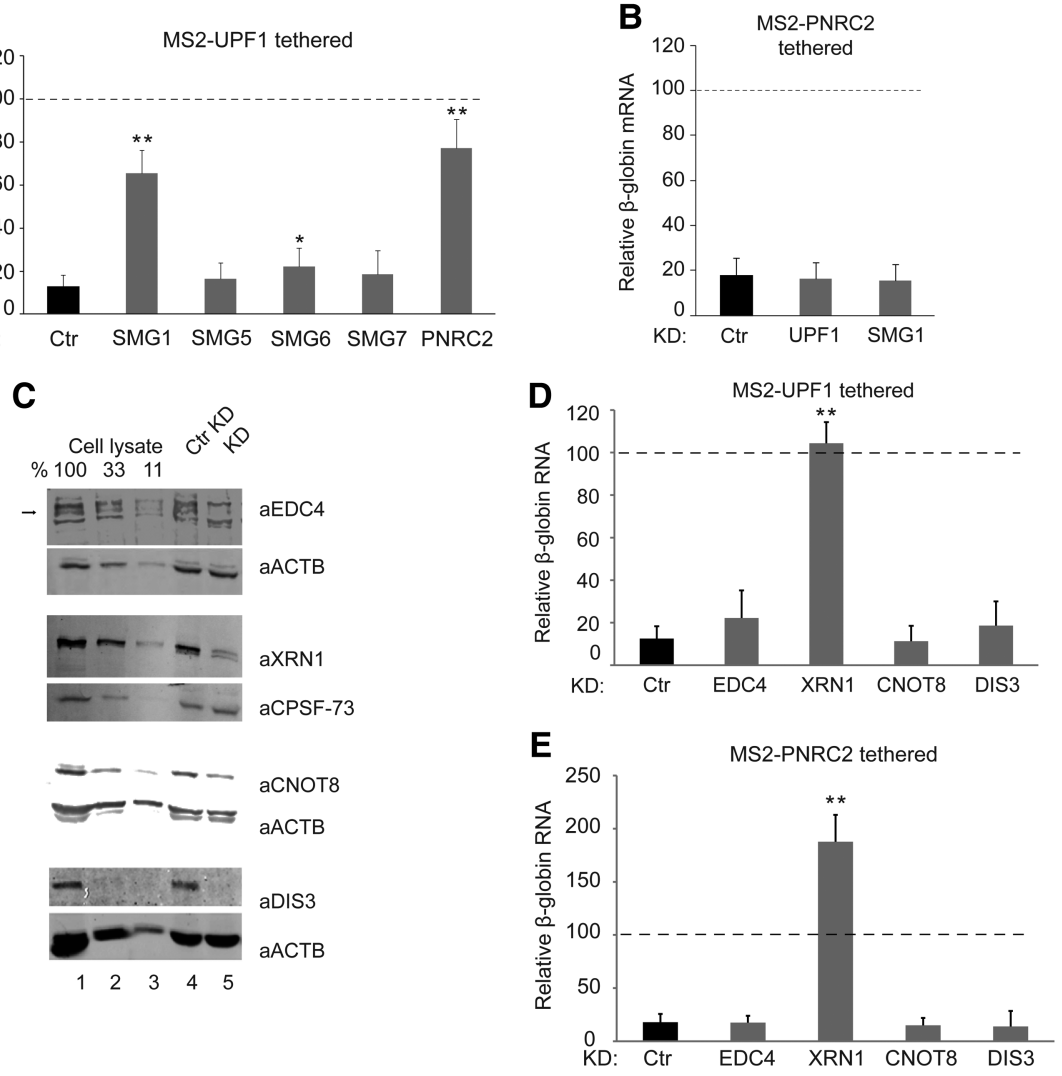

FIGURE 6. UPF1 and PNRC2 rely on XRN1 to induce reporter mRNA reduction in TFAs. $(A)$ TFA of MS2-UPF1, performed in cells with a knockdown (KD) of the factors indicated at the bottom of each panel. Ctr denotes cells expressing a control shRNA. (B) TFA of MS2-PNRC2 performed in cells with a UPF1 and SMG1 KD. (C) Western blots examining the knockdown efficiencies of the indicated proteins from samples used in the experiments shown in $D$ and $E$. Lanes $1-3$ show serial dilutions $(100 \%, 33 \%$, and $11 \%)$ of cell lysate from untransfected HeLa cells. Lanes 4 and 5 display the protein levels from cells expressing a control shRNA (Ctr KD) and the shRNA targeting the designated degradation factor (KD), respectively. The antibodies used are indicated at the right of each blot and the arrow signifies the band representing EDC4. Detection of Actin or CPSF-73 served as loading controls. $(D, E)$ MS2-UPF1 and MS2PNRC2 TFAs performed in cells depleted of degradation factors. The bar chart represents the relative $\beta$-globin reporter mRNA levels under control knockdown (Ctr) conditions (black bars) and the designated knockdown conditions (depleted factor indicated below each gray bar). The mean and SD of $>4$ independent experiments are shown, with $P$-values $\leq 0.01$ depicted as $\left(^{* *}\right)$ and $\leq$ 0.05 as $\left(^{*}\right)$.

Our results showing interactions between UPF1, PNRC2, decapping factors and XRN1 raised the question as to whether in the TFA, UPF1 might be inducing reporter mRNA degradation by direct decapping of the mRNA followed by XRN1-mediated $5^{\prime}-3^{\prime}$ exonucleolytic decay. Consistent with this hypothesis and somewhat unexpectedly considering that UPF1 tethering has been widely used as a surrogate to study the mechanism of NMD, tethered UPF1 did not require its downstream NMD effectors, SMG5, SMG6, or SMG7 to reduce reporter mRNA levels, but instead was strongly inhibited by the knockdowns of SMG1 and PNRC2 (Fig. 6A; Supplemental Table $\mathrm{S} 7$ for $P$-values and Supplemental Fig. S6A,B for SMG5 knockdown efficiency), suggesting that tethered UPF1 becomes phosphorylated by SMG1 and recruits the decapping complex and XRN1. Tethered

PNRC2 on the other hand did not depend on UPF1 or SMG1 to down-regulate the reporter mRNA (Fig. 6B; Supplemental Table S7 for $P$-values), which positions PNRC2 downstream from UPF1 and SMG1 in this degradation pathway. To further delineate the mechanism by which tethered UPF1 leads to the degradation of the reporter mRNA, we performed additional TFAs with tethered UPF1 or tethered PNRC2 in cells depleted for several well-characterized mRNA degradation factors. Specifically, we knocked down EDC4 (also known as Hedls), which is a decapping enhancer that has been shown to promote complex formation between DCP1A and DCP2 (Fenger-Gron et al. 2005; Chang et al. 2014), XRN1, which is the major $5^{\prime}-3^{\prime}$ exoribonuclease involved in mRNA decay (Arribas- 
Layton et al. 2013; Jonas et al. 2013), CNOT8 (also known as POP2), which has $3^{\prime}-5^{\prime}$ poly(A) exoribonuclease activity (Collart and Panasenko 2012), and DIS3 (also known as RRP44), which is the catalytic component of the RNA exosome complex and has both $3^{\prime}-5^{\prime}$ exonuclease (Dziembowski et al. 2007) as well as endonuclease (Lebreton et al. 2008; Schaeffer et al. 2009) activities and participates in a multitude of cellular RNA processing and degradation events. A fraction of each cell lysate was used to assess the knockdown efficiencies by western blotting (Fig. 6C). From the remainder of each cell lysate, we isolated total RNA and used RTqPCR to document the knockdowns also at the mRNA level (Supplemental Fig. S6C-F) and to measure the $\beta$-globin reporter mRNA levels, which are shown relative to the level in cells expressing LacZ-MS2 and normalized to GFP mRNA encoded on a cotransfected expression plasmid to account for differences in transfection efficiencies among the samples (Fig. 6D,E; Supplemental Table S7 for $P$-values). Our data revealed that the reduced reporter mRNA levels induced by MS2-UPF1 and MS2-PNRC2 tethering were both dependent on XRN1. Reporter mRNA bound by UPF1 or PNRC2 increased more than eightfold or 10-fold, respectively, in cells depleted of XRN1, while depletion of EDC4, CNOT8 and DIS3 did not significantly alter the $\beta$-globin mRNA levels compared to control cells (Fig. 6D, E). This indicates that the most dominant route of degradation induced by UPF1 artificially tethered to an mRNA is not by recruitment of NMD factors, but rather by interacting with PNRC2, which is dependent upon the presence of XRN1.

In summary, all of our data so far suggest that tethered UPF1 can recruit PNRC2 to induce decapping followed by mRNA degradation by the $5^{\prime}-3^{\prime}$ exonuclease XRN1 resulting in the reduced reporter mRNA levels observed in the TFA. Furthermore, since this occurs independently of SMG5, SMG6, or SMG7, it begs the question if and how well the UPF1 TFA mimics the process of NMD. Furthermore, the association of PNRC2 with XRN1 and decapping factors in conjunction with our lack of evidence for an interaction with SMG5 challenges the previous categorization of PNRC2 as a NMD factor.

\section{PNRC2 seems not to be required for NMD in vivo}

To directly test whether PNRC2 is needed for NMD, we used two well-characterized NMD reporter genes, TCR $\beta$ Ter68 and $\beta$-globin Ter39, and their respective PTC-free control constructs (designated WT) (Thermann et al. 1998; Mühlemann et al. 2001). In these classical NMD assays, UPF1 knockdown led to a 10 -fold increase of TCR $\beta$ Ter68 and $\beta$-globin Ter39 mRNA, while knockdown of PNRC2 had no effect on the mRNA levels of these two reporter genes (Fig. 7A,B; Supplemental Table S8 for $P$-values and Supplemental Fig. S7A,B for PNRC2 and UPF1 KD efficiencies). We also examined the effect of knocking down
UPF1, SMG5, SMG6, SMG7 and PNRC2 (Supplemental Fig. S7C,D) on the mRNA levels of the NMD reporter gene mini- $\mu$ Ter310 (Bühler et al. 2004) and its PTC-free version mini- $\mu$ WT (Fig. 7C,D; Supplemental Table S9 for $P$-values). Depletion of UPF1 and SMG6 led to an eightfold and 33-fold increase in mini- $\mu$ Ter310 mRNA, respectively, while knockdown of SMG5, SMG7 or PNRC2 alone had essentially no effect (Fig. 7C). Since there is evidence that in human cells SMG6-mediated endonucleolytic cleavage acts as the predominant mode of NMD in mammalian cells, while SMG5-SMG7-mediated deadenylation functions on the same target mRNAs merely as a backup system (Jonas et al. 2013; Metze et al. 2013; Boehm et al. 2014; Colombo et al. 2017), we reasoned that the PNRC2-dependent decay pathway might also be inferior to the SMG6 pathway. Therefore, we determined mRNA levels of mini$\mu$ Ter310 and mini- $\mu$ WT in cells where SMG5, SMG7 or PNRC2 was codepleted with SMG6. While codepletion of SMG6 with SMG5 or SMG7 inhibited NMD even more or similarly as SMG6 depletion alone, the increase of mini- $\mu$ Ter310 mRNA observed by codepletion of PNRC2 and SMG6 was in fact even less pronounced than in the SMG6 knockdown alone, most probably owing to the generally less efficient knockdown efficiency of the individual factors in the combined knockdowns. Furthermore, the combined knockdown of PNRC2 and SMG5 resulted in the same barely significant increase of mini- $\mu$ Ter310 mRNA as knockdown of each of these factors separately, indicating that they seem not to be involved in a common pathway. Finally, we wondered if PNRC2's function in NMD might be redundant to both the SMG6-dependent and the SMG5-SMG7-dependent pathway and thus we performed triple knockdowns of either SMG5, SMG6, and PNRC2, or SMG7, SMG6, and PNRC2. In these triple knockdowns, the mini- $\mu$ Ter310 mRNA levels increased two- to threefold more than in the respective double knockdowns (Fig. 7C). However, this increase was not PTC-specific and hence not caused by NMD, since mini- $\mu$ WT mRNA levels increased to a similar extent (Fig. 7D). Collectively, these results are consistent with the previously reported evidence for a redundancy between the SMG6-dependent and the SMG5-SMG7-dependent decay of NMD targets, and we could not find any evidence for the specific involvement of PNRC2 in degrading PTC-containing mRNAs, not even when we inhibited the more dominant SMG6-dependent mode of NMD.

Overall, we find no requirement for and thus probably no involvement of PNRC2 in NMD in HeLa cells for three different NMD reporters. On the other hand, we confirmed that UPF1, SMG6 and SMG5-SMG7 do feature in the decay of PTC-containing reporter $\mathrm{mRNAs}$ and that there appears to be a hierarchy between the predominant SMG6-mediated endonucleolytic cleavage induced mode of decay and the less active SMG5-SMG7-mediated decay pathway, which seems to function as a backup system. 

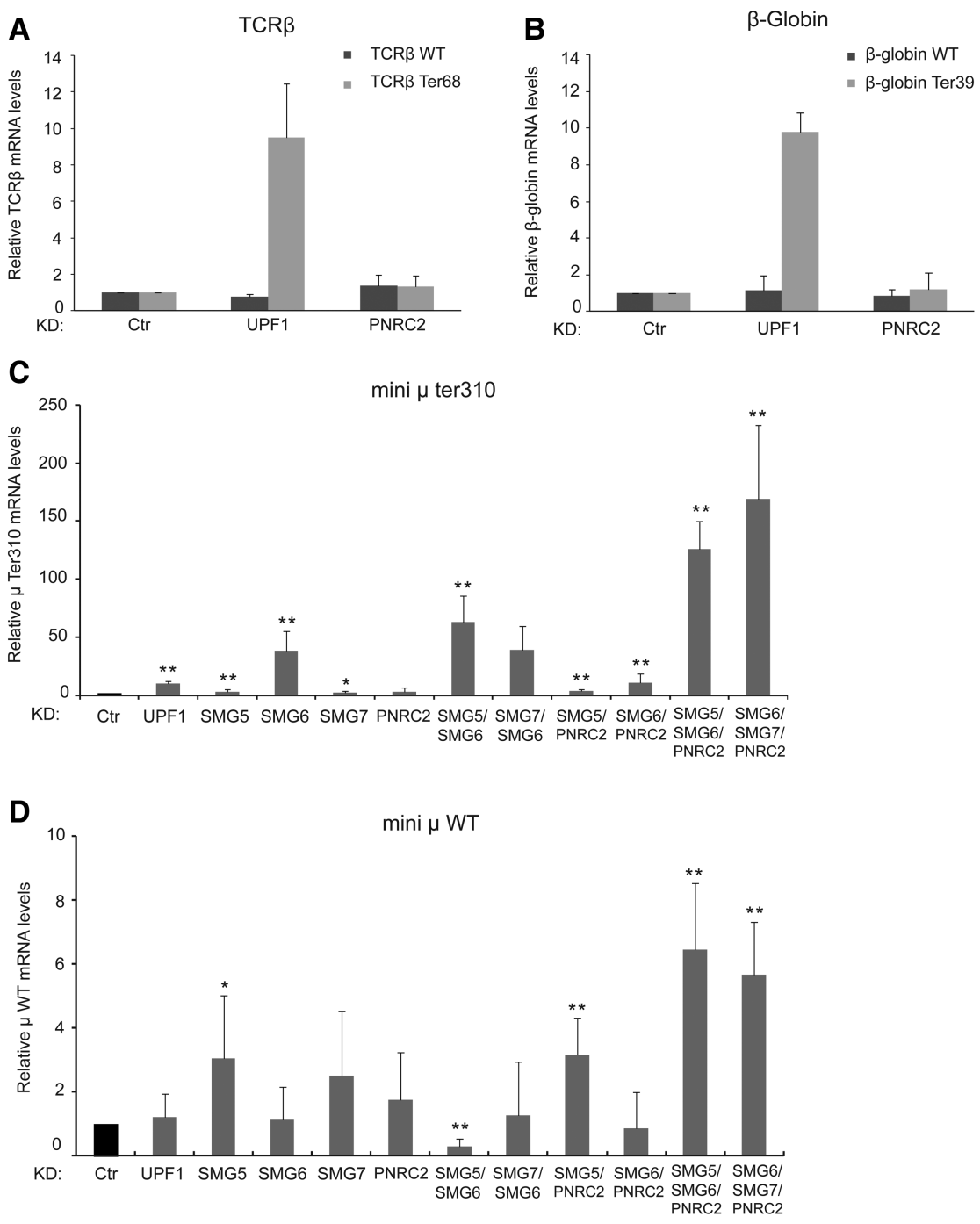

FIGURE 7. PNRC2 appears to be dispensable for NMD. $(A, B)$ HeLa cells were transfected with plasmids expressing either NMD insensitive WT TCR $\beta(A)$ or $\beta$-globin $(B)$ (black bars) or their PTC-containing counterparts (gray bars), along with pEGFP, and with plasmids expressing either a control shRNA (Ctr) or shRNAs targeting UPF1 or PNRC2. RT-qPCR analysis was used to measure the relative TCR $\beta$ or $\beta$-globin mRNA levels, normalized to EGFP mRNA levels. The levels of normalized NMD reporters in control knockdown cells were set to one to display fold changes. The mean and SD of three independent experiments are shown. $(C, D)$ HeLa cells were transfected with plasmids expressing mini- $\mu \operatorname{Ter} 310(C)$ or mini- $\mu$ WT $(D)$, along with pEGFP serving as a cotransfection control and with plasmids expressing either a control shRNA (Ctr, black bars) or shRNAs targeting the factors specified under each gray bar. Mini- $\mu$ mRNA levels, normalized to EGFP mRNA, were determined by RT-qPCR and are shown relative to the levels of the respective mini- $\mu$ mRNA in the control Ctr knockdown cells, which were set to one. Mean values and SD of $\geq 3$ independent experiments are represented. In all charts, $\left({ }^{* *}\right)$ signifies $P \leq 0.01$ and $\left({ }^{*}\right)$ denotes $P \leq 0.05$ as determined by Student's $t$-tests.

\section{DISCUSSION}

Using in vivo interaction mapping by yeast two-hybrid assays and immunoprecipitation experiments combined with various functional experiments, we investigated how SMG5, SMG7, and PNRC2 act downstream from UPF1 to promote the degradation of target mRNAs. Our results (Fig. 4A,B; Supplemental Figs. S4, S5) confirm the previously demon- strated interaction between SMG5 and SMG7 (Jonas et al. 2013) and show that the ability of tethered SMG5 to induce mRNA degradation depends on SMG7 (Fig. 2A), which is consistent with a study reporting that the $\mathrm{C}$ terminus of SMG7 recruits the CCR4-NOT deadenylase complex to the mRNA via an interaction with CNOT8 (Loh et al. 2013). Interestingly, tethered SMG5 appears to be able to induce reporter mRNA decay in two different ways. In the presence of SMG7, the SMG7/CCR4-NOT-dependent way is the preferred one and UPF1 is dispensable for this pathway (Fig. 2A), while the UPF1-PNRC2-dependent pathway (see below) appears to be used when the CCR4-NOT-binding C-terminal region of SMG7 is missing (Fig. 3). Altogether, our data in conjunction with evidence from previous studies (Unterholzner and Izaurralde 2004; Loh et al. 2013) suggest that one mRNA degradation pathway activated by P-UPF1 during mammalian NMD is the recruitment of the SMG5SMG7 heterodimer, which in turn leads to SMG7-mediated recruitment of the CCR4-NOT deadenylase complex. Furthermore, such a conclusion is also consistent with various early reports suggesting that deadenylation features in the decay of nonsense mRNAs (Chen and Shyu 2003; Lejeune et al. 2003).

In contrast, our data are inconsistent with a study reporting a SMG5PNRC2-mediated degradation pathway in mammalian NMD, which was reported to be dominant over the SMG5SMG7 pathway (Cho et al. 2013). We have not been able to detect the previously described interaction between SMG5 and PNRC2, neither by yeast two-hybrid assays nor by LC-MS/MS analysis of proteins coimmunoprecipitating with SMG5 or PNRC2 (Fig. 4; Supplemental Figs. S4, S5). Our results are in line with the pulldown experiments performed by Loh and colleagues who also failed to detect an interaction between SMG5 and PNRC2 (Loh et al. 2013). The association of PNRC2 with different decapping factors in IPs (EDC3, EDC4, DCP1A, DCP1B, and DDX6; Figs. 4B, 5) instead suggests that PNRC2 is most likely a constituent of the decapping machinery. Furthermore, in a small yeast mating screen, we observed PNRC2 interacting with DCP1, XRN1, the DCP1/ DCP2-associated Sm-like proteins LSM1, 2 and 6 (data not 
shown). Similarly, Cho and colleagues have also reported coprecipitation of DCP1A, DCP2, EDC4, and weakly of EDC3 with PNRC2 (Cho et al. 2013). Further challenging the reported SMG5-PNRC2-dependent decay pathway, the down-regulation of the reporter transcript induced by tethered SMG5 was not dependent on PNRC2 or UPF1 but instead was dependent on SMG7 (Figs. 2A, 3). Collectively, our data do not provide evidence for a physical or functional association between SMG5 and PNRC2. However, we cannot rule out that a SMG5-PNRC2-dependent mRNA degradation mode might exist in specific cell types or under specific conditions, but even if it did, it would be unlikely to rely on a direct interaction between PNRC2 and SMG5.

Nonetheless, this still leaves the question as to the role of PNRC2 in the TFA and in NMD. We found PNRC2 interacting with the N-terminal part of UPF1 (amino acids 1-271) in our yeast two-hybrid assays (Fig. 4A; Supplemental Fig. S5), which is consistent with previous pull-down experiments demonstrating that the $\mathrm{N}$-terminal conserved region (amino acids 1-72) of UPF1 is required for this interaction (Cho et al. 2009; Loh et al. 2013). However, unlike this study, we could not detect any requirement for the $\mathrm{C}$ terminus of UPF1 contributing to the interaction with PNRC2 in our yeast-two hybrid assays, most likely because the interaction in the $\mathrm{C}$ terminus of UPF1 involves phosphorylated SQ epitopes, which probably remain unphosphorylated in yeast (Yamashita 2013). In accordance with the observed physical interaction between UPF1 and PNRC2, tethered UPF1 required PNRC2 to reduce the reporter mRNA levels in the TFA (Fig. 6A), while tethered PNRC2 was not dependent on UPF1 (Fig. 6B), thus positioning PNRC2 downstream from UPF1 in this pathway. Notably, tethered UPF1 also required SMG1 for its activity (Fig. 6A) and both, PNRC2 and DCP1A copurified preferentially P-UPF1 (Fig. 5C,E), indicating that UPF1 must be phosphorylated by SMG1 to function in the TFA. Thus, our co-IP (Figs. 4C, 5) and TFA results (Fig. 6) collectively suggest that P-UPF1 recruits the decapping complex via PNRC2, which then promotes decapping of the reporter transcript followed by its XRN1-catalysed rapid 5' $-3^{\prime}$ exonucleolytic degradation. The exact role of PNRC2 in human mRNA decapping is not known, but PNRC2 interacts with DCP1A (Figs. 4C, 5C; Albers et al. 2005; Cho et al. 2009; Lai et al. 2012; Loh et al. 2013; Mugridge et al. 2016) and this interaction has been shown to stimulate the decapping activity of DCP2 by bridging the interaction between DCP1 and DCP2 (Lai et al. 2012), similar to EDC4, which also has been reported to bridge the DCP1-DCP2 interaction (Fenger-Gron et al. 2005; Chang et al. 2014). EDC4 can interact with DCP1, DCP2 and XRN1 in human cells and accordingly plays a pivotal role in mRNA decapping and the coordination of decapping to $5^{\prime}-3^{\prime}$ exonucleolytic decay by positioning XRN1 correctly for receiving decapped mRNAs (Braun et al. 2012; Chang et al. 2014). We have found that tethered PNRC2 does not need EDC4 but only XRN1, and that tethered UPF1 only requires SMG1, PNRC2 and
XRN1 to induce reporter decay (Fig. 6). Thus, it may be that PNRC2 is also a crucial mRNA decapping enhancer analogous to EDC4 and can act redundantly in this role with EDC4, or perhaps PNRC2 is working together with EDC4 since our mass spectrometry experiments detected PNRC2 copurifying with other known mRNA decapping enhancers as well as with DCP1 and DCP2 (Fig. 4C).

Unexpectedly, the UPF1-PNRC2-XRN1 mRNA degradation pathway observed in the TFA was not affected by knocking down SMG5, SMG6, or SMG7 (Fig. 6), raising the question if and to which extent the mRNA degradation process triggered by artificially tethering UPF1 to a reporter transcript reflects the process occurring during NMD. Therefore, it was important to test the requirement of PNRC2 on classical PTC-containing NMD reporter transcripts in vivo. PNRC2 knockdown, despite being as efficient as a UPF1 knockdown judged by the reduction of the respective mRNA levels (Supplemental Fig. S7), did not significantly increase the abundance of any of the three tested NMD reporters, while UPF1 knockdown caused an eight- to 20 -fold increase (Fig. 7). Given the previously reported redundancies between the SMG5-SMG7-dependent and the SMG6-dependent NMD pathways (Jonas et al. 2013; Metze et al. 2013; Colombo et al. 2017), we reasoned that possibly PNRC2-mediated RNA decay might not have been detected because of a similar redundancy. Therefore, we also depleted PNRC2 in combination with SMG6. Even under these conditions, we did not observe PNRC2 contributing to the reduction of the mini- $\mu$ Ter310 NMD reporter levels (Fig. 7C). A combined knockdown of PNRC2 and SMG5 also failed to inhibit NMD of the mini- $\mu$ Ter310 reporter construct, and the observed PNRC2-associated effect on mini- $\mu$ mRNA levels in triple knockdowns of PNRC2-SMG5SMG6 or PNRC2-SMG7-SMG6 were not PTC-specific (Fig. 7C,D). Altogether, these data indicate that PNRC2 is not involved in NMD of the mini- $\mu$ Ter310 reporter transcript in HeLa cells. We cannot rule out that PNRC2 may play a role in NMD of specific transcripts or other cell types.

Little is known concerning the function of SMG5 in NMD and our results have only revealed a little more insight. SMG5 knockdown had only a modest effect on the tested NMD reporter mRNAs (Fig. 7C and data not shown), whereas it robustly diminished the reporter mRNA levels in the TFA in a manner that was sensitive to SMG7 depletion (Figs. 2, 3). The dimerization with SMG7 was previously documented in vitro and by immunoprecipitation experiments (Anders et al. 2003; Ohnishi et al. 2003; Jonas et al. 2013) and here confirmed by yeast two-hybrid assays and mass spectrometry analyses (Fig. 4B; Supplemental Fig. S5). Furthermore, SMG5 coprecipitated UPF1 (Fig. 4B) and our yeast two-hybrid results showed that SMG5 can interact with the $\mathrm{C}$ terminus of UPF1, where the phosphorylated sites are located, but also hints at SMG5 alone being able to bind other parts of UPF1, too (Supplemental Fig. S5). Thus, altogether our data are consistent with SMG5 being an adaptor that 
facilitates the interaction between UPF1 and SMG7 during NMD, but further work is required to fully understand the role of SMG5 in NMD.

It has become very clear from our study that dissecting the role of UPF1 in NMD is particularly complicated by the evidence that UPF1 functions in additional cellular processes that appear to affect mRNA stability independent from its function in NMD (Kaygun and Marzluff 2005; Kim et al. 2005; Azzalin and Lingner 2006; Choe et al. 2014; Cho et al. 2015). The fact that UPF1 is involved in other mRNA degradation events independently of the NMD machinery became also evident in our TFAs (Fig. 6). As a consequence of UPF1's involvement in different RNA decay pathways, it should be noted that documenting a requirement for UPF1 is not sufficient to identify an RNA degradation pathway as NMD. Along the same lines, physical association with UPF1 is not sufficient to identify an mRNA as an NMD target, since recent studies revealed that UPF1 initially binds most mRNAs in the cell rather unspecifically and then selectively releases from mRNAs that are not degraded by NMD (Hurt et al. 2013; Zünd et al. 2013; Lee et al. 2015). The emerging picture is that UPF1 may have a scaffolding function and can assemble a multitude of different mRNPs tailored to various different degradation pathways.

In addition to the mechanistic aspects addressed here, our work clearly illustrates the need for an unambiguous operational definition of the term "nonsense-mediated mRNA decay" (NMD) in mammalian cells. Originally, this term was used to describe the rapid degradation of mRNAs with an open reading frame-interrupting PTC, and the key criteria to distinguish NMD from other RNA decay pathways was that the corresponding PTC-free transcript was not affected (Maquat 1995). When it was later discovered that even PTCfree mRNAs can be stabilized by inactivation of various NMD factors in human cells (Mendell et al. 2004; Viegas et al. 2007; Yepiskoposyan et al. 2011; Tani et al. 2012; Celik et al. 2017; Colombo et al. 2017), the defining criteria for NMD shifted away from cis-acting features of the target mRNAs toward the dependence of an mRNA destabilization event on the known trans-acting NMD factors. However, an NMD definition relying on the requirement for a set of trans-acting factors is problematic as it is based on the assumption that only one NMD mechanism exists that requires an identical set of trans-acting factors for all NMDtargeted mRNAs. Yet, this assumption could be incorrect, since there is evidence in mammalian systems for an EJCenhanced and an EJC-independent NMD pathway (Zhang et al. 1998; Rajavel and Neufeld 2001; Delpy et al. 2004; Bühler et al. 2006; Matsuda et al. 2007; Singh et al. 2008; Metze et al. 2013), as well as evidence for a UPF2-independent and a UPF3-independent pathway (Gehring et al. 2005; Chan et al. 2007; Metze et al. 2013) and redundancy between SMG6-dependent and SMG7-dependent NMD (Colombo et al. 2017). It is not known if these reported heterogeneities in mammalian NMD actually reflect the existence of multi- ple, mechanistically distinct NMD pathways, or if there is one common pathway with different factors being more rate-limiting for some substrates than for others. Future work further elucidating the spatial and temporal molecular interactions among all known NMD factors will ultimately reveal the long desired detailed mechanistic understanding of NMD.

\section{MATERIALS AND METHODS}

\section{Plasmids}

The plasmids expressing MS2-UPF1 and MS2-PNRC2 are explained elsewhere (Lykke-Andersen et al. 2000; Cho et al. 2009), respectively. Plasmids encoding SMG5-MS2-HA and SMG7 (isoform 1)MS2-HA were generated by inserting the corresponding cDNAs into the NheI site of pCMV-MS2-HA (Eberle et al. 2008). The two NheI sites in the SMG5 open reading frame were abolished by creating silent mutations using site-directed mutagenesis as described earlier (Nicholson et al. 2012).

To generate pcDNA3-FLAG-SMG7(iso2)-RNAiR, silent mutations in the region targeted by our shRNA assay were introduced to pcDNA3-FLAG-SMG7 isoform 2 (Colombo et al. 2017) using site mutagenesis as outlined earlier (Nicholson et al. 2012). The plasmids encoding the SMG7 deletion constructs were generated by PCR amplification using primer pairs introducing XhoI and ApaI sites to nucleotides $1-2514(\Delta \mathrm{C})$ and $1-1895(\Delta \mathrm{PC})$ of SMG7 isoform 2. The amplified constructs were then sub-cloned into XhoI-ApaI cut pcDNA3-FLAG-SMG7(iso2)-RNAiR.

The plasmids expressing decapping factors DCP1A and DCP2 (pcDNA3-FLAG-Myc-DCP1A and pcDNA3-FLAG-Myc-DCP2, respectively) and the plasmid expressing EGFP-tagged XRN1 were previously described (Lykke-Andersen and Wagner 2005; Braun et al. 2012). The plasmid expressing pcDNA3-HA-UPF1(isoform 2)-RNAiR was previously explained (Nicholson et al. 2014). To obtain the pcDNA3-HA-EGFP plasmid, the EGFP ORF was ligated into pcDNA3-HA downstream from the HA tag.

The plasmids expressing SMG5 and SMG7 (isoform 1) fused to GAL4 DNA-AD were generated by PCR amplification using plasmids with NdeI and XhoI restriction sites and subsequent sub-cloning into pCR 2.1-TOPO vector (Life Technologies). In the TOPO vector, the NdeI site in the open reading frame of SMG7 was destroyed by site-directed mutagenesis creating a silent point mutation. From here, the SMG5 and SMG7 cDNAs were inserted into NdeIXhol cut pGADT7 (Clontech). Plasmid GADT7-UPF1 and pGADT7-PNRC2 were made by PCR amplification using primers with incorporated NdeI and BamHI sites, sub-cloning of the amplicons into pCR 2.1-TOPO and pSC-A-amp/kan (Agilent Technologies) vectors, respectively, cut out and inserted into a NdeI-BamHI cut pGADT7. Plasmid GBKT7-SMG5 and pGBKT7-PNRC2 were generated by excising the SMG5 and PNRC2 fragments from the vectors described above and inserting them into the NdeI-NotI or NdeI-BamHI sites of pGBKT7 (Clontech), respectively. The pGBKT7-SMG5 and pGADT7-SMG7 mutants were attained by introducing specific point mutations in the equivalent wild-type plasmids using site-directed mutagenesis as described earlier (Nicholson et al. 2012). pGBKT7-UPF1 (isoform 2) and all its derivatives have been previously described (Nicholson et al. 2014). 
The plasmids encoding the $\beta$-globin Ter39, the TCR $\beta$ Ter68 and the mini- $\mu$ Ter310 NMD reporter constructs are described in Thermann et al. (1998), Mühlemann et al. (2001), and Bühler et al. (2004). The oligonucleotide sequences used to create the pSUPuro plasmids are documented in the Supplemental Information.

\section{Tethered function, rescue, and NMD assays}

Cells were seeded into six-well plates and DNA cotransfections were carried out using Lipofectamine 2000 (Life Technologies) according to the manufacturer's guidelines. In all TFAs, $100 \mathrm{ng}$ pEGFP-C3, $100 \mathrm{ng}$ pc $\beta$-globin-6xMS2bs and $1 \mu \mathrm{g}$ plasmid expressing SMG5MS2-HA, MS2-PNRC2, MS2-UPF1 or LacZ-MS2-HA or HAMS2-LacZ, were used. When combined with knockdowns, an additional $400 \mathrm{ng}$ of pSUPuro plasmid(s) was cotransfected. For the rescue assays in Figure 3, an additional $800 \mathrm{ng}$ of the SMG7-WT ${ }^{\mathrm{R}}$, SMG7- $\Delta C^{\mathrm{R}}$, or SMG7- $\Delta \mathrm{PC}^{\mathrm{R}}$ constructs were cotransfected in the respective samples. In Figure 7, $100 \mathrm{ng}$ of $\operatorname{mini}-\mu$, TCR $\beta$, or $\beta$-globin reporter plasmids, $100 \mathrm{ng}$ pmCMV-pEGFP-C3, along with 400 ng pSUPuro plasmid were cotransfected. The remainder of the knockdown protocol, during which protein and RNA samples were always taken from the same sample, was as previously explained (Nicholson et al. 2012). Quantitative PCR assays are catalogued in the Supplemental Information section. To examine protein levels, cell lysates corresponding to 35,000 whole-cell equivalents per lane were separated on 6\%-15\% SDS-PAGE, and the proteins were transferred to a PVDF membrane (Westran Clear Signal, GE Healthcare) and probed with the specified primary antibodies and appropriate fluorophore-coupled secondary antibodies. Fluorescent signals were captured using the Odyssey Infrared Imaging System (LI-COR Biosciences). The full directory of all antibodies used in this study can be found in the Supplemental Tables S10 and S11.

\section{Yeast two-hybrid $\beta$-galactosidase plate assays}

Two hundred and fifty nanograms of the pGADT7 and pGBKT7 plasmids were cotransformed into Mav99 cells (Vidal et al. 1996) according to the high-efficiency LiOAc/single-stranded carrier DNA (Clontech)/PEG method of transformation (Gietz et al. 1992), and the yeast two hybrid $\beta$-galactosidase plate assays were performed as described in Nicholson et al. (2014).

\section{MS2-immunoprecipitation followed by mass spectrometry}

HeLa cells were seeded in to $10 \mathrm{~cm}$ dishes, and the next day cotransfections with either $10 \mu \mathrm{g}$ pCMV-SMG5-MS2-HA, pCMV-MS2PNRC2, or pCMV-HA-MS2-LacZ were carried out using Lipofectamine 2000. For the entire immunoprecipitation, gloves were worn to minimize keratin contamination, and low protein-Lobind tubes $1.5 \mathrm{~mL}$ (Eppendorf) were used. Two days post transfection, the cells were harvested and incubated at $3 \times 10^{4}$ cells $/ \mu \mathrm{L}$ in hypotonic gentle lysis buffer (HGLB: $10 \mathrm{mM}$ Tris-HCl pH 7.5, $10 \mathrm{mM} \mathrm{NaCl}, 2 \mathrm{mM}$ EDTA, $0.5 \%$ Triton X-100 freshly supplemented with $2 \times$ Halt protease [Thermo Scientific] and $20 \mu \mathrm{g} / \mathrm{mL}$ RNase A [Sigma-Aldrich] creating $\mathrm{HGLB}++)$. The lysate was centrifuged to collect the cell debris, the cleared lysate was retained and the salt concentration was adjusted to $150 \mathrm{mM} \mathrm{NaCl}$. Five micrograms of anti-Enterobacterio Phage MS2 Coat Protein Antibody (Millipore, ABE76) was incubated with the lysate at $4^{\circ} \mathrm{C}$ for 1.5 h. $35 \mu \mathrm{L} / \mathrm{IP}$ of Dynabeads Protein G (Life Technologies) were washed twice with wash buffer (TBS, $0.05 \%$ Tergitol-type NP-40) and once with high salt $\mathrm{HGLB}++(150 \mathrm{mM} \mathrm{NaCl})$. The antibody/lysate mixture was combined with the cleared beads and incubated at $4^{\circ} \mathrm{C}$ for $1 \mathrm{~h}$. An input sample (3\%) was taken preceding this step and afterwards, the unbound fraction was retained. The beads were washed once with high salt HGLB++ and then three times with a wash buffer lacking any detergent $(10 \mathrm{mM}$ Tris- $\mathrm{HCl} \mathrm{pH} 7.5$ and $150 \mathrm{mM} \mathrm{NaCl})$. The final wash buffer was removed, the beads were collected in a fresh tube and the mass spectrometry procedure was performed by the Proteomics \& Mass Spectrometry Core Facility at the University of Bern as described in Joncourt et al. (2014), except the trypsin digestion was performed directly on the beads. Semi-quantitative protein abundance was assessed using the calculated protein match score summation (PMSS). Specifically, the total protein scores were calculated by adding up all peptide $z$-scores belonging to the same protein. Then the PMSS values were corrected by multiplication by the molecular weight of the native protein (considering no posttranslational modifications) as given on the EXPASY website (www.expasy.org) and division by the number of theoretically observable peptides, which was defined as peptides following the trypsin cleavage rules (C-terminal to lysine and arginine, not if proline at $\mathrm{P}_{1}{ }^{\prime}$ position) with zero or one missed cleavage and having a molecular mass between 720 and $3000 \mathrm{kDa}$ (Colinge et al. 2004). This whole experiment was carried out twice independently.

\section{MS2, GFP, and FLAG immunoprecipitations}

HEK293T cells were seeded in to $15 \mathrm{~cm}$ dishes and the next day cotransfected with $11 \mu \mathrm{g}$ pCMV-HA-MS2-LacZ, $11 \mu \mathrm{g}$ pCMVMS2-UPF1, $11 \mu \mathrm{g}$ pCMV-MS2-HA-PNRC2, $6 \mu \mathrm{g}$ pcDNA3-HA$\mathrm{UPF}^{\mathrm{R}}, 6 \mu \mathrm{g}$ pcDNA3-HA-EGFP, $26 \mu \mathrm{g}$ pT7-EGFP-C1-HsXRN1, $3 \mu \mathrm{g}$ pCMV6-mGADPH-myc-FLAG, $7.5 \mu \mathrm{g}$ pcDNA3-FLAGMyc-DCP1A and $26 \mu \mathrm{g}$ pcDNA3-FLAG-Myc-DCP2 in pairs according to the schemes in Figure 5. Lipofectamine 2000 was used for transfections and cells were harvested after $48 \mathrm{~h}$. The remainder of the immunoprecipitation protocol is as explained above with some minor alterations or additions. Namely, $2 \times 10^{7}$ cells were collected for lysis in $1 \mathrm{~mL}$ HGLB supplemented with $1 \times$ protease inhibitor cocktail (Biotool), the lysates were incubated with either $4 \mu \mathrm{g}$ of anti-MS2 (Millipore, ABE76) or $4 \mu \mathrm{g}$ of anti-FLAG(M2), the GFP immunoprecipitation lysates were incubated directly with $5 \mu \mathrm{L} / \mathrm{IP}$ Dynabeads m270 Epoxy (Thermo Scientific) coupled with anti-GFP, according to the manufacturer's protocol $(8 \mu \mathrm{g} / \mathrm{mg}$ of beads), and where indicated, RNase A (Sigma) was added to a final concentration of $200 \mu \mathrm{g} / \mathrm{mL}$ in IP wash buffer (TBS $0.05 \%$ NP40 supplemented with protease inhibitor) and incubated at RT for 30 min prior to elution. At the final wash step the beads were collected in a fresh tube and eluted in $30 \mu \mathrm{L}$ of NuPAGE LDS sample buffer (Thermo Fisher Scientific). Protein samples from input and immunoprecipitations (100\% of the IP samples) were incubated at $75^{\circ} \mathrm{C}$ for $10 \mathrm{~min}$ before loading on the NuPAGE gels (Thermo Fisher Scientific). After electrophoresis, the proteins were transferred to an Optitran BA-S 85 reinforced nitrocellulose membrane (Whatman $\mathrm{GmbH}$ ), followed by immunoblotting as outlined above. 


\section{SUPPLEMENTAL MATERIAL}

Supplemental material is available for this article.

\section{ACKNOWLEDGMENTS}

We are very grateful to Jens Lykke-Andersen (UCSD, USA) for various plasmids and antibodies, to Yoon Ki Kim (Korea University, Seoul, Republic of Korea) for the MS2-PNRC2 expression plasmid and anti-PNRC2 antibody, to Berndt Müller (University of Aberdeen, UK) for the pGBKT7 and pGADT7 plasmids, to Chris Sanderson (University of Liverpool, UK) for kindly donating his repertoire of fusion proteins transformed into the PJ69-4a and PJ69-4a yeast strains, to Bertrand Seraphin (IGBMC, France) for kindly sharing the anti-CNOT8 antibody, to Torben Heick Jensen (Aarhus University, Denmark) for the Anti-RRP44 antibody, to MarcDavid Ruepp for the pCMV6-mGADPH-myc-FLAG (Origene MR204934), to Seline Zürcher, Stefan Reber, and Nicola Rusca for aiding in the cloning of various plasmids. Finally, we thank Manfred Heller and Sophie Lagache Braga (University of Bern, Switzerland) for performing the mass spectrometric analyses. This work was supported by funds from the European Research Council (ERC-StG 207419), the Swiss National Science Foundation (SNSF) (grants 31003A-127614, -143717, and -162986), the National Center of Competence in Research RNA \& Disease (funded by the SNSF), the Novartis Foundation for Biomedical Research and the canton Bern, and the Nomis Foundation.

Received August 21, 2017; accepted January 8, 2018.

\section{REFERENCES}

Albers M, Kranz H, Kober I, Kaiser C, Klink M, Suckow J, Kern R, Koegl M. 2005. Automated yeast two-hybrid screening for nuclear receptor-interacting proteins. Mol Cell Proteomics 4: 205-213.

Anders KR, Grimson A, Anderson P. 2003. SMG-5, required for C. elegans nonsense-mediated mRNA decay, associates with SMG-2 and protein phosphatase 2A. EMBO J 22: 641-650.

Arribas-Layton M, Wu D, Lykke-Andersen J, Song H. 2013. Structural and functional control of the eukaryotic mRNA decapping machinery. Biochim Biophys Acta 1829: 580-589.

Azzalin CM, Lingner J. 2006. The double life of UPF1 in RNA and DNA stability pathways. Cell Cycle 5: 1496-1498.

Bhattacharya A, Czaplinski K, Trifillis P, He F, Jacobson A, Peltz SW. 2000. Characterization of the biochemical properties of the human Upf1 gene product that is involved in nonsense-mediated mRNA decay. RNA 6: 1226-1235.

Boehm V, Haberman N, Ottens F, Ule J, Gehring NH. 2014. 3' UTR length and messenger ribonucleoprotein composition determine endocleavage efficiencies at termination codons. Cell Rep 9: $555-568$.

Braun JE, Truffault V, Boland A, Huntzinger E, Chang CT, Haas G, Weichenrieder O, Coles M, Izaurralde E. 2012. A direct interaction between DCP1 and XRN1 couples mRNA decapping to $5^{\prime}$ exonucleolytic degradation. Nat Struct Mol Biol 19: 1324-1331.

Bühler M, Paillusson A, Mühlemann O. 2004. Efficient downregulation of immunoglobulin $\mu$ mRNA with premature translation-termination codons requires the $5^{\prime}$-half of the VDJ exon. Nucleic Acids Res 32: 3304-3315.

Bühler M, Steiner S, Mohn F, Paillusson A, Mühlemann O. 2006. EJCindependent degradation of nonsense immunoglobulin- $\mu$ mRNA depends on 3' UTR length. Nat Struct Mol Biol 13: 462-464.
Celik A, Baker R, He F, Jacobson A. 2017. High-resolution profiling of NMD targets in yeast reveals translational fidelity as a basis for substrate selection. RNA 23: 735-748.

Chakrabarti S, Bonneau F, Schüssler S, Eppinger E, Conti E. 2014. Phospho-dependent and phospho-independent interactions of the helicase UPF1 with the NMD factors SMG5-SMG7 and SMG6. Nucleic Acids Res 42: 9447-9460.

Chan WK, Huang L, Gudikote JP, Chang YF, Imam JS, MacLean JA II, Wilkinson MF. 2007. An alternative branch of the nonsense-mediated decay pathway. EMBO J 26: 1820-1830.

Chang CT, Bercovich N, Loh B, Jonas S, Izaurralde E. 2014. The activation of the decapping enzyme DCP2 by DCP1 occurs on the EDC4 scaffold and involves a conserved loop in DCP1. Nucleic Acids Res 42: 5217-5233.

Chen CY, Shyu AB. 2003. Rapid deadenylation triggered by a nonsense codon precedes decay of the RNA body in a mammalian cytoplasmic nonsense-mediated decay pathway. Mol Cell Biol 23: 4805-4813.

Chiu SY, Serin G, Ohara O, Maquat LE. 2003. Characterization of human Smg5/7a: a protein with similarities to Caenorhabditis elegans SMG5 and SMG7 that functions in the dephosphorylation of Upf1. RNA 9: 77-87.

Cho H, Kim KM, Kim YK. 2009. Human proline-rich nuclear receptor coregulatory protein 2 mediates an interaction between mRNA surveillance machinery and decapping complex. Mol Cell 33: 75-86.

Cho H, Han S, Choe J, Park SG, Choi SS, Kim YK. 2013. SMG5-PNRC2 is functionally dominant compared with SMG5-SMG7 in mammalian nonsense-mediated mRNA decay. Nucleic Acids Res 41: 1319-1328.

Cho H, Park OH, Park J, Ryu I, Kim J, Ko J, Kim YK. 2015. Glucocorticoid receptor interacts with PNRC2 in a ligand-dependent manner to recruit UPF1 for rapid mRNA degradation. Proc Natl Acad Sci 112: E1540-E1549.

Choe J, Ahn SH, Kim YK. 2014. The mRNP remodeling mediated by UPF1 promotes rapid degradation of replication-dependent histone mRNA. Nucleic Acids Res 42: 9334-9349.

Clerici M, Mourão A, Gutsche I, Gehring NH, Hentze MW, Kulozik A, Kadlec J, Sattler M, Cusack S. 2009. Unusual bipartite mode of interaction between the nonsense-mediated decay factors, UPF1 and UPF2. EMBO J 28: 2293-2306.

Colinge J, Chiappe D, Lagache S, Moniatte M, Bougueleret L. 2004. Differential proteomics via probabilistic peptide identification scores. Anal Chem 77: 596-606.

Collart MA, Panasenko OO. 2012. The Ccr4-Not complex. Gene 492: 42-53.

Colombo M, Karousis ED, Bourquin J, Bruggmann R, Mühlemann O. 2017. Transcriptome-wide identification of NMD-targeted human mRNAs reveals extensive redundancy between SMG6- and SMG7mediated degradation pathways. RNA 23: 189-201.

Czaplinski K, Ruiz-Echevarria MJ, Paushkin SV, Han X, Weng Y, Perlick HA, Dietz HC, Ter-Avanesyan MD, Peltz SW. 1998. The surveillance complex interacts with the translation release factors to enhance termination and degrade aberrant mRNAs. Genes Dev 12: $1665-1677$.

Delpy L, Sirac C, Magnoux E, Duchez S, Cogné M. 2004. RNA surveillance down-regulates expression of nonfunctional $\kappa$ alleles and detects premature termination within the last $\kappa$ exon. Proc Natl Acad Sci 101: 7375-7380.

Dziembowski A, Lorentzen E, Conti E, Séraphin B. 2007. A single subunit, Dis3, is essentially responsible for yeast exosome core activity. Nat Struct Mol Biol 14: 15-22.

Eberle AB, Stalder L, Mathys H, Orozco RZ, Mühlemann O. 2008. Posttranscriptional gene regulation by spatial rearrangement of the $3^{\prime}$ untranslated region. PLoS Biol 6: e92.

Eberle AB, Lykke-Andersen S, Mühlemann O, Jensen TH. 2009. SMG6 promotes endonucleolytic cleavage of nonsense mRNA in human cells. Nat Struct Mol Biol 16: 49-55.

Fatscher T, Boehm V, Weiche B, Gehring NH. 2014. The interaction of cytoplasmic poly(A)-binding protein with eukaryotic initiation 
factor $4 \mathrm{G}$ suppresses nonsense-mediated mRNA decay. RNA 20: $1579-1592$.

Fenger-Gron M, Fillman C, Norrild B, Lykke-Andersen J. 2005. Multiple processing body factors and the ARE binding protein TTP activate mRNA decapping. Mol Cell 20: 905-915.

Fields S, Song O. 1989. A novel genetic system to detect protein-protein interactions. Nature 340: 245-246.

Finley RL Jr, Brent R. 1994. Interaction mating reveals binary and ternary connections between Drosophila cell cycle regulators. Proc Natl Acad Sci 91: 12980-12984.

Flury V, Restuccia U, Bachi A, Mühlemann O. 2014. Characterization of phosphorylation- and RNA-dependent UPF1 interactors by quantitative proteomics. J Proteome Res 13: 3038-3053.

Fukuhara N, Ebert J, Unterholzner L, Lindner D, Izaurralde E, Conti E. 2005. SMG7 is a 14-3-3-like adaptor in the nonsense-mediated mRNA decay pathway. Mol Cell 17: 537-547.

Gehring NH, Kunz JB, Neu-Yilik G, Breit S, Viegas MH, Hentze MW, Kulozik AE. 2005. Exon-junction complex components specify distinct routes of nonsense-mediated mRNA decay with differential cofactor requirements. Mol Cell 20: 65-75.

Gietz D, St. Jean A, Woods RA, Schiestl RH. 1992. Improved method for high efficiency transformation of intact yeast cells. Nucleic Acids Res 20: 1425 .

Glavan F, Behm-Ansmant I, Izaurralde E, Conti E. 2006. Structures of the PIN domains of SMG6 and SMG5 reveal a nuclease within the mRNA surveillance complex. EMBO J 25: 5117-5125.

He F, Li X, Spatrick P, Casillo R, Dong S, Jacobson A. 2003. Genomewide analysis of mRNAs regulated by the nonsense-mediated and $5^{\prime}$ to $3^{\prime}$ mRNA decay pathways in yeast. Mol Cell 12: 1439-1452.

Houseley J, Tollervey D. 2009. The many pathways of RNA degradation. Cell 136: 763-776.

Hug N, Longman D, Cáceres JF. 2016. Mechanism and regulation of the nonsense-mediated decay pathway. Nucleic Acids Res 44: 1483-1495.

Huntzinger E, Kashima I, Fauser M, Saulière J, Izaurralde E. 2008. SMG6 is the catalytic endonuclease that cleaves mRNAs containing nonsense codons in metazoan. RNA 14: 2609-2617.

Hurt JA, Robertson AD, Burge CB. 2013. Global analyses of UPF1 binding and function reveal expanded scope of nonsense-mediated mRNA decay. Genome Res 23: 1636-1650.

Isken O, Kim YK, Hosoda N, Mayeur GL, Hershey JW, Maquat LE. 2008. Upf1 phosphorylation triggers translational repression during nonsense-mediated mRNA decay. Cell 133: 314-327.

Ivanov PV, Gehring NH, Kunz JB, Hentze MW, Kulozik AE. 2008. Interactions between UPF1, eRFs, PABP and the exon junction complex suggest an integrated model for mammalian NMD pathways. EMBO J 27: 736-747.

Jonas S, Weichenrieder O, Izaurralde E. 2013. An unusual arrangement of two 14-3-3-like domains in the SMG5-SMG7 heterodimer is required for efficient nonsense-mediated mRNA decay. Genes Dev 27: 211-225.

Joncourt R, Eberle AB, Rufener SC, Mühlemann O. 2014. Eukaryotic initiation factor $4 \mathrm{G}$ suppresses nonsense-mediated mRNA decay by two genetically separable mechanisms. PLoS One 9: e104391.

Karousis ED, Nasif S, Mühlemann O. 2016. Nonsense-mediated mRNA decay: novel mechanistic insights and biological impact. Wiley Interdiscip Rev RNA 7: 661-682.

Kashima I, Yamashita A, Izumi N, Kataoka N, Morishita R, Hoshino S, Ohno M, Dreyfuss G, Ohno S. 2006. Binding of a novel SMG-1Upf1-eRF1-eRF3 complex (SURF) to the exon junction complex triggers Upf1 phosphorylation and nonsense-mediated mRNA decay. Genes Dev 20: 355-367.

Kaygun H, Marzluff WF. 2005. Regulated degradation of replication-dependent histone mRNAs requires both ATR and Upf1. Nat Struct Mol Biol 12: 794-800.

Kervestin S, Jacobson A. 2012. NMD: a multifaceted response to premature translational termination. Nat Rev Mol Cell Biol 13: 700-712.

Kim YK, Furic L, Desgroseillers L, Maquat LE. 2005. Mammalian Staufen1 recruits Upf1 to specific mRNA $3^{\prime}$ UTRs so as to elicit mRNA decay. Cell 120: 195-208.
Lai T, Cho H, Liu Z, Bowler MW, Piao S, Parker R, Kim YK, Song H. 2012. Structural basis of the PNRC2-mediated link between mRNA surveillance and decapping. Structure 20: 2025-2037.

Lebreton A, Tomecki R, Dziembowski A, Séraphin B. 2008. Endonucleolytic RNA cleavage by a eukaryotic exosome. Nature 456: 993-996.

Lee SR, Pratt GA, Martinez FJ, Yeo GW, Lykke-Andersen J. 2015. Target discrimination in nonsense-mediated mRNA decay requires Upf1 ATPase activity. Mol Cell 59: 413-425.

Lejeune F, Li X, Maquat LE. 2003. Nonsense-mediated mRNA decay in mammalian cells involves decapping, deadenylating, and exonucleolytic activities. Mol Cell 12: 675-687.

Lewis BP, Green RE, Brenner SE. 2003. Evidence for the widespread coupling of alternative splicing and nonsense-mediated mRNA decay in humans. Proc Natl Acad Sci 100: 189-192.

Ling SHM, Qamra R, Song H. 2011. Structural and functional insights into eukaryotic mRNA decapping. Wiley Interdiscip Rev RNA 2: 193-208.

Llorca O. 2012. Structural insights into nonsense-mediated mRNA decay (NMD) by electron microscopy. Curr Opin Struct Biol 23: 161-167.

Loh B, Jonas S, Izaurralde E. 2013. The SMG5-SMG7 heterodimer directly recruits the CCR4-NOT deadenylase complex to mRNAs containing nonsense codons via interaction with POP2. Genes Dev 27: $2125-2138$.

Lykke-Andersen J. 2002. Identification of a human decapping complex associated with hUpf proteins in nonsense-mediated decay. Mol Cell Biol 22: 8114-8121.

Lykke-Andersen S, Jensen TH. 2015. Nonsense-mediated mRNA decay: an intricate machinery that shapes transcriptomes. Nat Rev Mol Cell Biol 16: 665-677.

Lykke-Andersen J, Wagner E. 2005. Recruitment and activation of mRNA decay enzymes by two ARE-mediated decay activation domains in the proteins TTP and BRF-1. Genes Dev 19: 351-361.

Lykke-Andersen J, Shu MD, Steitz JA. 2000. Human Upf proteins target an mRNA for nonsense-mediated decay when bound downstream of a termination codon. Cell 103: 1121-1131.

Maquat LE. 1995. When cells stop making sense: effects of nonsense codons on RNA metabolism in vertebrate cells. RNA 1: 453-465.

Matsuda D, Hosoda N, Kim YK, Maquat LE. 2007. Failsafe nonsensemediated mRNA decay does not detectably target eIF4E-bound mRNA. Nat Struct Mol Biol 14: 974-979.

Mendell JT, Sharifi NA, Meyers JL, Martinez-Murillo F, Dietz HC. 2004. Nonsense surveillance regulates expression of diverse classes of mammalian transcripts and mutes genomic noise. Nat Genet 36: 1073-1078.

Metze S, Herzog VA, Ruepp MD, Mühlemann O. 2013. Comparison of EJC-enhanced and EJC-independent NMD in human cells reveals two partially redundant degradation pathways. RNA 19: 1432-1448.

Mugridge JS, Ziemniak M, Jemielity J, Gross JD. 2016. Structural basis of mRNA-cap recognition by Dcp1-Dcp2. Nat Struct Mol Biol 23: 987-994.

Mühlemann O, Mock-Casagrande CS, Wang J, Li S, Custódio N, Carmo-Fonseca M, Wilkinson MF, Moore MJ. 2001. Precursor RNAs harboring nonsense codons accumulate near the site of transcription. Mol Cell 8: 33-43.

Nicholson P, Yepiskoposyan H, Metze S, Zamudio Orozco R, Kleinschmidt N, Mühlemann O. 2010. Nonsense-mediated mRNA decay in human cells: mechanistic insights, functions beyond quality control and the double-life of NMD factors. Cell Mol Life Sci 67: 677-700.

Nicholson P, Joncourt R, Mühlemann O. 2012. Analysis of nonsensemediated mRNA decay in mammalian cells. Curr Protoc Cell Biol 55: 27.4.1-27.4.61.

Nicholson P, Josi C, Kurosawa H, Yamashita A, Mühlemann O. 2014. A novel phosphorylation-independent interaction between SMG6 and UPF1 is essential for human NMD. Nucleic Acids Res 42: 9217-9235.

Ohnishi T, Yamashita A, Kashima I, Schell T, Anders KR, Grimson A, Hachiya T, Hentze MW, Anderson P, Ohno S. 2003. 
Phosphorylation of hUPF1 induces formation of mRNA surveillance complexes containing hSMG-5 and hSMG-7. Mol Cell 12: $1187-1200$.

Okada-Katsuhata Y, Yamashita A, Kutsuzawa K, Izumi N, Hirahara F, Ohno S. 2012. N- and C-terminal Upf1 phosphorylations create binding platforms for SMG-6 and SMG-5:SMG-7 during NMD. Nucleic Acids Res 40: 1251-1266.

Page MF, Carr B, Anders KR, Grimson A, Anderson P. 1999. SMG-2 is a phosphorylated protein required for mRNA surveillance in Caenorhabditis elegans and related to Upf1p of yeast. Mol Cell Biol 19: $5943-5951$.

Rajavel KS, Neufeld EF. 2001. Nonsense-mediated decay of human HEXA mRNA. Mol Cell Biol 21: 5512-5519.

Rehwinkel J, Letunic I, Raes J, Bork P, Izaurralde E. 2005. Nonsense-mediated mRNA decay factors act in concert to regulate common mRNA targets. RNA 11: 1530-1544.

Schaeffer D, Tsanova B, Barbas A, Reis FP, Dastidar EG, SanchezRotunno M, Arraiano CM, van Hoof A. 2009. The exosome contains domains with specific endoribonuclease, exoribonuclease and cytoplasmic mRNA decay activities. Nat Struct Mol Biol 16: 56-62.

Schoenberg DR, Maquat LE. 2012. Regulation of cytoplasmic mRNA decay. Nat Rev Genet 13: 246-259.

Schweingruber C, Rufener SC, Zünd D, Yamashita A, Mühlemann O. 2013. Nonsense-mediated mRNA decay-mechanisms of substrate mRNA recognition and degradation in mammalian cells. Biochim Biophys Acta 1829: 612-623.

Silva AL, Ribeiro P, Inácio A, Liebhaber SA, Romão L. 2008. Proximity of the poly(A)-binding protein to a premature termination codon inhibits mammalian nonsense-mediated mRNA decay. RNA 14: 563-576.

Singh G, Rebbapragada I, Lykke-Andersen J. 2008. A competition between stimulators and antagonists of Upf complex recruitment governs human nonsense-mediated mRNA decay. PLoS Biol 6: e111.

Stansfield I, Jones KM, Kushnirov VV, Dagkesamanskaya AR, Poznyakovski AI, Paushkin SV, Nierras CR, Cox BS, TerAvanesyan MD, Tuite MF. 1995. The products of the SUP45 (eRF1) and SUP35 genes interact to mediate translation termination in Saccharomyces cerevisiae. EMBO J 14: 4365-4373.
Tani H, Imamachi N, Salam KA, Mizutani R, Ijiri K, Irie T, Yada T, Suzuki Y, Akimitsu N. 2012. Identification of hundreds of novel UPF1 target transcripts by direct determination of whole transcriptome stability. RNA Biol 9: 1370-1379.

Thermann R, Neu-Yilik G, Deters A, Frede U, Wehr K, Hagemeier C, Hentze MW, Kulozik AE. 1998. Binary specification of nonsense codons by splicing and cytoplasmic translation. EMBO J 17: 3484-3494.

Unterholzner L, Izaurralde E. 2004. SMG7 acts as a molecular link between mRNA surveillance and mRNA decay. Mol Cell 16: 587-596.

Vidal M, Brachmann RK, Fattaey A, Harlow E, Boeke JD. 1996. Reverse two-hybrid and one-hybrid systems to detect dissociation of protein-protein and DNA-protein interactions. Proc Natl Acad Sci 93: 10315-10320.

Viegas MH, Gehring NH, Breit S, Hentze MW, Kulozik AE. 2007. The abundance of RNPS1, a protein component of the exon junction complex, can determine the variability in efficiency of the nonsense mediated decay pathway. Nucleic Acids Res 35: 4542-4551.

Yamashita A. 2013. Role of SMG-1-mediated Upf1 phosphorylation in mammalian nonsense-mediated mRNA decay. Genes Cells 18: 161-175.

Yepiskoposyan H, Aeschimann F, Nilsson D, Okoniewski M, Mühlemann O. 2011. Autoregulation of the nonsense-mediated mRNA decay pathway in human cells. RNA 17: 2108-2118.

Zhang J, Sun X, Qian Y, Maquat LE. 1998. Intron function in the nonsense-mediated decay of $\beta$-globin mRNA: indications that premRNA splicing in the nucleus can influence mRNA translation in the cytoplasm. RNA 4: 801-815.

Zhou D, Shen R, Ye JJ, Li Y, Tsark W, Isbell D, Tso P, Chen S. 2008. Nuclear receptor coactivator PNRC2 regulates energy expenditure and adiposity. J Biol Chem 283: 541-553.

Zhouravleva G, Frolova L, Le Goff X, Le Guellec R, Inge-Vechtomov S, Kisselev L, Philippe M. 1995. Termination of translation in eukaryotes is governed by two interacting polypeptide chain release factors, eRF1 and eRF3. EMBO J 14: 4065-4072.

Zünd D, Gruber AR, Zavolan M, Mühlemann O. 2013. Translation-dependent displacement of UPF1 from coding sequences causes its enrichment in 3' UTRs. Nat Struct Mol Biol 20: 936-943. 

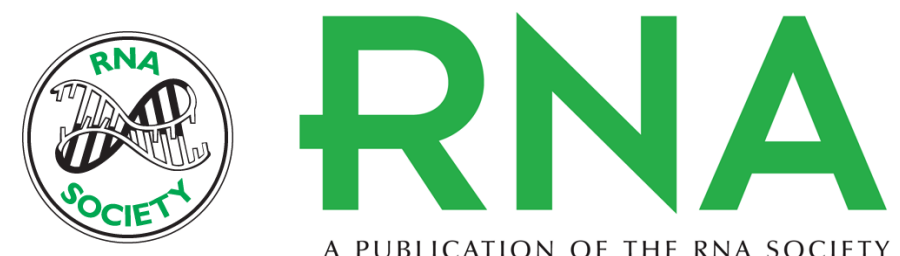

A PUBLICATION OF THE RNA SOCIETY

\section{Dissecting the functions of SMG5, SMG7, and PNRC2 in nonsense-mediated mRNA decay of human cells}

Pamela Nicholson, Asimina Gkratsou, Christoph Josi, et al.

RNA 2018 24: 557-573 originally published online January 18, 2018

Access the most recent version at doi:10.1261/rna.063719.117

\section{Supplemental http://rnajournal.cshlp.org/content/suppl/2018/01/18/rna.063719.117.DC1 Material}

References This article cites 94 articles, 35 of which can be accessed free at: http://rnajournal.cshlp.org/content/24/4/557.full.html\#ref-list-1

Open Access Freely available online through the RNA Open Access option.

Creative This article, published in $R N A$, is available under a Creative Commons License Commons (Attribution-NonCommercial 4.0 International), as described at License http://creativecommons.org/licenses/by-nc/4.0/.

Email Alerting Receive free email alerts when new articles cite this article - sign up in the box at the Service top right corner of the article or click here.

To subscribe to $R N A$ go to:

http://rnajournal.cshlp.org/subscriptions 